\title{
Elastic symmetries of defective crystals
}

\author{
G. P. Parry
}

Received: date / Accepted: date

\begin{abstract}
I construct discrete and continuous crystal structures that are compatible with a given choice of dislocation density tensor, and (following Mal' cev) provide a canonical form for these discrete structures. The symmetries of the discrete structures extend uniquely to symmetries of corresponding continuous structures - I calculate these symmetries explicitly for a particular choice of dislocation density tensor and deduce corresponding constraints on energy functions which model defective crystals.
\end{abstract}

Keywords Crystals · Defects $\cdot$ Lie groups

Mathematics Subject Classification (2000) 74A20 $\cdot 74 \mathrm{E} 25$

\section{Introduction}

I consider solid crystals with uniform distribution of defects (in the sense that the corresponding dislocation density tensor is constant in space) and treat issues related to the symmetry properties of these materials using the theory of Lie groups. Specifically I focus on the particular case where the lattice components of the dislocation density tensor, denoted $S_{a b}$, have the form

$$
S_{a b}=\lambda p_{a} p_{b}, \quad \lambda, p_{a} \in \mathbb{R}, a, b=1,2,3,
$$

where the corresponding Lie group is nilpotent. This appears to be the simplest case where one can construct discrete and continuous structures compatible with a given choice of $S \equiv\left(S_{a b}\right)$. When (1) holds (in fact, when $S$ is restricted a little further), I note that results of Mal'cev [1] provide

- a canonical form for the discrete structures that have the dislocation density tensor (ddt) specified,

- a connection between the symmetries of these discrete structures and the symmetries of a corresponding continuous structure.

G. P. Parry

School of Mathematical Sciences, University of Nottingham, Nottingham, NG7 2RD, UK.

E-mail: gareth.parry@nottingham.ac.uk 
The paper is a continuation of work in Cermelli and Parry [2], Elzanowski and Parry [3], Parry [4],[5], whose purpose is to generalize work on the mechanics of perfect crystals $(S=0)$ to allow for the existence of defects $(S \neq 0)$. I focus, throughout the paper, on how concepts related to the crystallography of perfect crystals transfer and generalize to continuous and discrete models of materials with defects. In particular, the discrete structures that Mal'cev considers are the analogues of the simple lattices that are prominent in traditional treatments of the case $S=0$, and the symmetry properties of these structures may be discussed in a manner closely related to the method of traditional crystallography.

I work with Davini's model of solid crystals [6] (see also Davini and Parry [7], [8]), where the kinematical state of the crystal is given by the prescription of three smooth linearly independent vector fields $\ell_{1}(\cdot), \ell_{2}(\cdot), \ell_{3}(\cdot)$ in a domain which one may presume to be $\mathbb{R}^{3}$. In this context, the dislocation density tensor is defined by

$$
S_{a b}=\frac{\nabla \wedge \mathbf{d}_{a} \cdot \mathbf{d}_{b}}{\mathbf{d}_{1} \cdot \mathbf{d}_{2} \wedge \mathbf{d}_{3}}, \quad a, b=1,2,3
$$

when the fields $\mathbf{d}_{1}(\cdot), \mathbf{d}_{2}(\cdot), \mathbf{d}_{3}(\cdot)$ are dual to the 'lattice vector' fields $\boldsymbol{\ell}_{1}(\cdot), \boldsymbol{\ell}_{2}(\cdot), \boldsymbol{\ell}_{3}(\cdot)$. Nominally, the quantity on the right hand side of (2) is evaluated at some point $\mathbf{x} \in \mathbb{R}^{3}$, but in Parry [4], [5], [9], reasons (based on the motivation for the work) are given to consider just the case where the right hand side of (2) is independent of $\mathbf{x}$, for each $a, b,=1,2,3$. So I confine attention to the case $S=$ constant here, and note that when $S$ is constant, the values of $S$ are not arbitrary (derive an expression for $\nabla \wedge \mathbf{d}_{a}$ from (2), take the divergence and obtain a constraint on the values of $S$ ). However the choice of $S$ that is made in (1) is consistent with this constraint.

It is the assumption that $S$ is constant that leads to the connection with the theory of Lie groups. Indeed the assumption turns out to be an integrability condition which guarantees that the first order partial differential system

$$
\boldsymbol{\ell}_{a}(\boldsymbol{\psi}(\mathbf{x}, \mathbf{y}))=\nabla_{1} \boldsymbol{\psi}(\mathbf{x}, \mathbf{y}) \boldsymbol{\ell}_{a}(\mathbf{x}), \quad a=1,2,3,
$$

for the unknown function $\psi$ (where the fields $\ell_{1}(\cdot), \ell_{2}(\cdot), \ell_{3}(\cdot)$ are as above, and are given, and where $\nabla_{1} \psi(\cdot, \cdot)$ denotes the gradient of $\psi$ with respect to its first argument) has a solution. Moreover it follows that

$$
\boldsymbol{\psi}(\boldsymbol{\psi}(\mathbf{x}, \mathbf{y}), \mathbf{z})=\boldsymbol{\psi}(\mathbf{x}, \boldsymbol{\psi}(\mathbf{y}, \mathbf{z}))
$$

that one can prescribe

$$
\psi(\mathbf{x}, \mathbf{0})=\psi(\mathbf{0}, \mathbf{x})=\mathbf{x},
$$

and that, for each $\mathbf{x}$, there exists an element $\mathbf{x}^{-1}$ such that

$$
\psi\left(\mathbf{x}, \mathrm{x}^{-1}\right)=\psi\left(\mathrm{x}^{-1}, \mathrm{x}\right)=\mathbf{0} .
$$

Thus, $\boldsymbol{\psi}$ can be viewed as a group composition (or, multiplication) function. For the choice of $S$ given by (1), it turns out that the domain of $\boldsymbol{\psi}$ is $\mathbb{R}^{3} \times \mathbb{R}^{3}$. So, given lattice vector fields $\ell_{1}(\cdot), \ell_{2}(\cdot), \ell_{3}(\cdot)$, with corresponding constant ddt of the form (1), one arrives at a particular Lie group composition function by solving (3). Let $G$ denote the relevant Lie group.

Now the ddt is an elastic invariant (see Davini and Parry [7]), so that there are many choices of lattice vector fields which have given ddt of the form (1). So if only 
$S_{a b}=\lambda p_{a} p_{b}, a, b=1,2,3$, is given, there are many corresponding Lie group composition functions $\boldsymbol{\psi}$, and many corresponding choices of lattice vector fields. It will be a central task in the paper to choose, from amongst this class of 'equivalent' composition functions, one which has useful properties so far as simplifying the geometry of the relevant discrete structure concerned.

To motivate what is to be done, I recall well known facts in the case $S=0$. There, the simple discrete structure that has 'useful properties' is a perfect lattice, $L$ say, and the class of 'equivalent' structures consists of elastic images of the points of $L$. The choice of $\boldsymbol{\psi}$ that produces the simple lattice $L$ is $\boldsymbol{\psi}(\mathbf{x}, \mathbf{y})=\mathbf{x}+\mathbf{y}$, for (3) gives then that $\boldsymbol{\ell}_{a}(\mathbf{x}+\mathbf{y})=\boldsymbol{\ell}_{a}(\mathbf{x}), \mathbf{x}, \mathbf{y} \in \mathbb{R}^{3}, a=1,2,3$, which implies that $\boldsymbol{\ell}_{a}(\mathbf{x})=\boldsymbol{\ell}_{a}(\mathbf{0}) \equiv \boldsymbol{\ell}_{a}$ say, $a=1,2,3$. Thus the fields $\ell_{1}(\cdot), \ell_{2}(\cdot), \ell_{3}(\cdot)$ may be chosen to be translation invariant (i.e. 'constant') in the case $S=0$. The relevant Lie group is $\mathbb{R}^{3}$ with addition as group operation, and the perfect lattices are the (only) discrete subgroups of this Lie group. (This is where perfect lattices enter the picture, in the case $S=0$. They are the only discrete subgroups of the Lie groups obtained by solving (3) for the composition function $\psi$ when the fields $\ell_{a}(\cdot), a=1,2,3$, are translation invariant. It is worth emphasizing that, a priori, the perfect lattices play no role in the case $S \neq 0$ - in that case, one has to solve (3) for $\boldsymbol{\psi}$, where fields $\boldsymbol{\ell}_{a}(\cdot), a=1,2,3$, are such that (2) holds with $S \neq 0$ constant, and find the discrete subgroups of the Lie group which has composition function $\psi$. These discrete subgroups will be the analogues of the perfect lattices, in the case $S \neq 0$ ).

Let

$$
L=\left\{\mathbf{x}: \mathbf{x}=n_{a} \ell_{a}, \quad n_{a} \in \mathbb{Z}, \quad a=1,2,3\right\}
$$

be a particular perfect (or 'simple') lattice, where the summation convention operates on repeated indices in (4) and throughout, except where stated otherwise. Discussions of the symmetries of $L$ derive from the bijections of $L$ that preserve addition - let $\phi: L \rightarrow L$ be such a bijection. Since $\phi(L)=L$ and $\left\{\ell_{1}, \ell_{2}, \ell_{3}\right\}$ is a basis of $L, \phi\left(\ell_{a}\right)=m_{a b} \ell_{b}, a, b,=1,2,3$, for some matrix $m \equiv\left(m_{a b}\right)$ whose elements are integers. By the presumed additivity $\phi\left(n_{a} \ell_{a}\right)=n_{a} \phi\left(\ell_{a}\right)$, so from $\phi(L)=$ $L, L=\left\{\mathbf{x}: \mathbf{x}=n_{a} \phi\left(\ell_{a}\right), n_{a} \in \mathbb{Z}, a=1,2,3\right\}$ and therefore $\phi\left(\ell_{1}\right), \phi\left(\ell_{2}\right), \phi\left(\ell_{3}\right)$ is a basis of $L$. Then $\ell_{a}=n_{a b} \phi\left(\ell_{b}\right)$, for some matrix of integers $n \equiv\left(n_{a b}\right)$. It follows that $\operatorname{det}(m) \operatorname{det}(n)=1$ (where $\operatorname{det}(\cdot)$ denotes the determinant), and so one has the result that $\phi$ corresponds to a matrix $m \in G L_{3}(\mathbb{Z})$ via the relation $\phi\left(\ell_{a}\right)=m_{a b} \ell_{b}$. Next, $\phi$ extends uniquely to a smooth bijection of $\mathbb{R}^{3}$ that preserves addition : for let $\boldsymbol{\theta}$ be such a bijection and suppose $\boldsymbol{\theta}\left(n_{a} \ell_{a}\right)=\phi\left(n_{a} \ell_{a}\right), n_{a} \in \mathbb{Z}$. Then one argues that $\boldsymbol{\theta}\left((r / s) \boldsymbol{\ell}_{a}\right)=(r / s) \boldsymbol{\theta}\left(\boldsymbol{\ell}_{a}\right)$ by additivity, where $r$ and $s$ are integers, and that $\boldsymbol{\theta}\left(t \boldsymbol{\ell}_{a}\right)=$ $t \boldsymbol{\theta}\left(\boldsymbol{\ell}_{a}\right), t \in \mathbb{R}$ by smoothness. So $\boldsymbol{\theta}\left(t_{a} \ell_{a}\right)=t_{a} \boldsymbol{\theta}\left(\boldsymbol{\ell}_{a}\right)=t_{a} \boldsymbol{\phi}\left(\boldsymbol{\ell}_{a}\right), t_{a} \in \mathbb{R}, a=1,2,3$, defines $\boldsymbol{\theta}$ on $\mathbb{R}^{3}-\boldsymbol{\theta}$ is a linear mapping uniquely determined by its values on a basis of $L$. This last property is not particularly useful in the case $S=0$ (it seems to me), but it will transpire that an analogous result holds in the case $S \neq 0$ (the result is that automorphisms of the discrete structures, which are particular kinds of subgroup of the Lie group $G$ determined by $S$, extend uniquely to automorphisms of $G$ ), and that result is very useful, by way of contrast.

In the body of the paper, I show how the statements of the last two paragraphs generalize to the case $S \neq 0$, and extend material presented in Parry [5]. I have already intimated that the analogues of the perfect lattices $L$ will turn out to be subgroups of the Lie group $G$ obtained by solving (3) for the composition function $\boldsymbol{\psi}$ (if the fields $\ell_{a}(\cdot), a=1,2,3$ are given), and stated that, if only $S$ is given, there is an arbitrariness 
in the choice of $G$ which derives from the fact that $S$ is an elastic invariant. In [1], Mal'cev discusses uniform discrete subgroups $D$ of $G$ (see later for the definition of uniform discrete subgroups - it is a different use of the term 'uniform' to that which occurs in the first sentence of the introduction. No confusion should occur as a result of the ambiguity.) I show in section 4 that these uniform discrete subgroups are just those which are discussed in Cermelli and Parry [2], Parry [5], and revisit the motivation for the assertion that the natural generalization of the perfect lattices $L$, in the case $S=0$, are the discrete subgroups of $G$, in the case $S \neq 0$. Mal'cev shows that, if $D$ is a uniform discrete subgroup of a nilpotent Lie group $G$, then $D$ has a canonical basis in the sense that there exist elements $\ell_{1}, \ell_{2}, \ell_{3} \in D$ such that

$$
D=\left\{\mathbf{g}: \mathbf{g}=\ell_{1}^{m_{1}} \ell_{2}^{m_{2}} \ell_{3}^{m_{3}} ; m_{1}, m_{2}, m_{3} \in \mathbb{Z}\right\},
$$

where the meaning of terms like $\boldsymbol{\ell}_{1} \boldsymbol{\ell}_{2}$, which appear in (8), is $\boldsymbol{\ell}_{1} \boldsymbol{\ell}_{2}=\boldsymbol{\psi}\left(\boldsymbol{\ell}_{1}, \boldsymbol{\ell}_{2}\right)$. The elements $\ell_{1}, \ell_{2}, \ell_{3}$ satisfy other conditions besides. Comparing (7) and (8), one sees that there is a close analogy between the perfect lattices $L \subset \mathbb{R}^{3}$ and the uniform discrete subgroups $D \subset G$. (There is a further constraint on $S$, in addition to the requirement that it has the form (1), if a nilpotent Lie group $G$ is to possess uniform discrete subgroups). Mal'cev also shows that, if $D$ is given and $\ell_{1}, \ell_{2}, \ell_{3}$ is a canonical basis, then there is a system of one parameter subgroups of $G$, denoted $\mathbf{x}_{1}(t), \mathbf{x}_{2}(t), \mathbf{x}_{3}(t)$ (i.e. subgroups $\left\{\mathbf{x}_{i}(t), t \in \mathbb{R}\right\}$ of $G$ such that $\mathbf{x}_{i}(t+s)=\boldsymbol{\psi}\left(\mathbf{x}_{i}(t), \mathbf{x}_{i}(s)\right), t, s \in \mathbb{R}, i=1,2,3$, no summation on $i$ ) with the following properties:

$$
\ell_{i}=\mathbf{x}_{i}(1), \quad i=1,2,3 ;
$$

each element of $G$ is expressible in the form

$$
\mathbf{x}_{1}\left(t_{1}\right) \mathbf{x}_{2}\left(t_{2}\right) \mathbf{x}_{3}\left(t_{3}\right) \equiv \boldsymbol{\psi}\left(\boldsymbol{\psi}\left(\mathbf{x}_{1}\left(t_{1}\right), \mathbf{x}_{2}\left(t_{2}\right)\right), \mathbf{x}_{3}\left(t_{3}\right)\right)
$$

for some $t_{1}, t_{2}, t_{3} \in \mathbb{R}$; and some other conditions besides. (In the case $S=0$, the elements $\ell_{1}, \ell_{2}, \ell_{3}$ represent elements of a basis of $L$, the one parameter groups $\mathbf{x}_{1}(\cdot), \mathbf{x}_{2}(\cdot), \mathbf{x}_{3}(\cdot)$ are straight lines through $\mathbf{0}, \boldsymbol{\ell}_{1}=\mathbf{x}_{1}(1) ; \mathbf{0}, \ell_{2}=\mathbf{x}_{2}(1) ; \mathbf{0}, \boldsymbol{\ell}_{3}=\mathbf{x}_{3}(1)$. Any element of $\mathbb{R}^{3}$ can be represented as a sum of three vectors, each vector taken from one of the three straight lines.) Moreover, it is a fact that any automorphism of $D$ extends uniquely to a smooth automorphism of $G$ - this is useful because it implies that the set of 'symmetries' of $D$ is a subset of the set of symmetries of $G$, if one understands by the word symmetry a one to one mapping that preserves the group multiplication in $D, G$ respectively. Mal'cev proves these results in generality, for nilpotent groups of arbitrary finite dimensionality - I use only the three dimensional versions of his results here.

Now notice that, in the case $S=0$, the relation $\phi\left(\ell_{a}\right)=m_{a b} \ell_{b}, m \in G L_{3}(\mathbb{Z})($ which is the basis for discussion of point groups, lattice groups, etc., in crystallography) derives from the additivity of the group operation $\psi(\mathbf{x}, \mathbf{y})=\mathbf{x}+\mathbf{y}$. Thus the relation involves making a choice in the class of Lie groups related to ddt $S=0$-it chooses the group where the group operation is addition. It turns out that a corresponding choice must be made in the case $S \neq 0$, in order to obtain a convincing analogue of this relation. Let $\mathfrak{g}$ be the Lie algebra corresponding to $G$ ( $\mathfrak{g}$ is determined just by $S$ ). $\mathfrak{g}$ is a vector space $\left(\mathbb{R}^{3}\right.$, here) with Lie bracket providing an antisymmetric bilinear operation on pairs of elements (there is a simple connection between the Lie bracket and the continuum mechanical construction of the Burgers vectors, given pairs of lattice vector fields). It is important to recognize that each Lie group related to a given ddt $S$ has 
the same Lie algebra $\mathfrak{g}$. Also, importantly, it transpires that one can introduce a multiplication operation between pairs of elements of $\mathfrak{g}$ such that $\mathfrak{g}$, with this operation, becomes a Lie group, which I denote $J$. (One uses the group multiplication in $G$ to define a corresponding operation in $J$, but the resulting operation is independent of the choice of $G$ ). Group multiplication in $J$ corresponds to the Campbell-Baker-Hausdorff formula. It turns out that the one parameter subgroups in $J$ are straight lines (through the origin) and one deduces that the automorphisms of $J$ are linear mappings ('homogeneous deformations'). It follows, by Mal'cev's results, that the automorphisms of $D \subset J$ are likewise linear mappings, and this makes the tasks of finding those automorphisms explicitly rather easier than it would otherwise have been. The generalization of the relation $\phi\left(\ell_{a}\right)=m_{a b} \ell_{b}, m \in G L_{3}(\mathbb{Z})$, is discussed in section 5 below.

In the body of the paper I discuss these results in detail, having first recalled the elements of Lie theory that are required for that discussion, in particular I calculate the automorphisms of an arbitrary uniform discrete $D \subset J$ explicitly, for $S$ of the form (1) with the elements of $S$ rational. I note also that there is a difference between the automorphisms of $D$ and the set of 'global symmetries' of $D$ introduced in Parry[5]. The former preserve the value of $S$, the 'global symmetries' do not necessarily do so. Those symmetries of $D$ that preserve $S$ extend to symmetries of $G$, and so they represent (restrictions of) elastic deformations - so I explain briefly how one can have elastic and inelastic symmetries of a set of points in this context.

\section{Lie groups and algebras}

\subsection{Generalities}

A Lie group $G$ is a group with the structure of a manifold, such that the group multiplication function $\psi: G \times G \rightarrow G$ is smooth. It will be sufficient for our purposes to consider groups $G$ such that an element $\mathbf{x} \in G$ is uniquely specified by three real numbers $x_{1}, x_{2}, x_{3}$ (called the coordinates of $\mathbf{x}$ ), and I shall write $\mathbf{x}=x_{i} \mathbf{e}_{i}$, where $\left\{\mathbf{e}_{1}, \mathbf{e}_{2}, \mathbf{e}_{3}\right\}$ is a basis of $\mathbb{R}^{3}$ as recognition of this fact. The group multiplication function $\psi$ satisfies the relations (4),(5),(6) above, where the coordinates of the group identity elements are chosen to be zero. In the context of the paper, attention may be and is restricted to Lie groups $G$ which are connected and simply connected.

As an alternative notation for the product of group elements $\mathbf{x}, \mathbf{y}$, which is $\boldsymbol{\psi}(\mathbf{x}, \mathbf{y})$, I shall often write

$$
\mathrm{xy} \equiv \psi(\mathbf{x}, \mathbf{y})
$$

Let $(\mathbf{x}, \mathbf{y})$ denote the commutator of two group elements:

$$
(\mathbf{x}, \mathbf{y}) \equiv \mathbf{x}^{-1} \mathbf{y}^{-1} \mathbf{x y} .
$$

It is a standard calculation that the quadratic term in the Taylor expansion of $(\mathbf{x}, \mathbf{y})$ is $\gamma(\mathbf{x}, \mathbf{y})$ (cf. Gorbatsevich, Onishchik, Vinberg [10]), where

$$
\gamma(\mathbf{x}, \mathbf{y}) \equiv C_{i j k} x_{j} y_{k} \mathbf{e}_{i}
$$

(with $\mathbf{y}=y_{i} \mathbf{e}_{i}$ ), and

$$
C_{i j k} \equiv\left(\left.\frac{\partial^{2} \psi_{i}}{\partial x_{j} \partial y_{k}}(\mathbf{x}, \mathbf{y})\right|_{\mathbf{x}=\mathbf{y}=\mathbf{0}}-\left.\frac{\partial^{2} \psi_{i}}{\partial x_{k} \partial y_{j}}(\mathbf{x}, \mathbf{y})\right|_{\mathbf{x}=\mathbf{y}=\mathbf{0}}\right)
$$


The function $\gamma$ provides a bilinear form $\gamma: \mathbb{R}^{3} \times \mathbb{R}^{3} \rightarrow \mathbb{R}^{3}$ which is antisymmetric and satisfies the Jabobi identity in the sense that

$$
C_{i j k} C_{j r s}+C_{i j r} C_{j s k}+C_{i j s} C_{j k r}=0,
$$

by virtue of the associativity of $\boldsymbol{\psi},(4)$. For our purposes, the vector space $\mathbb{R}^{3}$, with the operation $[\cdot, \cdot]: \mathbb{R}^{3} \times \mathbb{R}^{3} \rightarrow \mathbb{R}^{3}$ defined by

$$
[\mathbf{x}, \mathbf{y}]=\gamma(\mathbf{x}, \mathbf{y})
$$

is the Lie algebra of the group $G$, and $[\cdot, \cdot]$ is called the Lie bracket. The Lie bracket satisfies

$$
[\mathbf{x},[\mathbf{y}, \mathbf{z}]]+[\mathbf{y},[\mathbf{z}, \mathbf{x}]]+[\mathbf{z},[\mathbf{x}, \mathbf{y}]]=\mathbf{0},
$$

from (13), (15), (16). The constants $C_{i j k}$ which define the form $\gamma$ are called the structure constants of the Lie algebra.

Vector fields $\boldsymbol{\nu}(\cdot)$ defined on $G$ which satisfy

$$
\boldsymbol{\nu}(\boldsymbol{\psi}(\mathbf{x}, \mathbf{y}))=\nabla_{1} \boldsymbol{\psi}(\mathbf{x}, \mathbf{y}) \boldsymbol{\nu}(\mathbf{x}),
$$

are said to be right invariant on $G$, so the lattice vector fields which satisfy (3) are right invariant. Let $\boldsymbol{\nu}(\cdot)$ be right invariant on $G$ and consider the integral curve of $\boldsymbol{\nu}(\cdot)$ through the point $\mathbf{x}_{0}$ : this is the set $\{\mathbf{x}(t): t \in \mathbb{R}\}$ which represents the solution of

$$
\frac{d \mathbf{x}}{d t}(t)=\boldsymbol{\nu}(\mathbf{x}(t)), \mathbf{x}(0)=\mathbf{x}_{0}, \quad t \in \mathbb{R} .
$$

It is a standard result that, if $\mathbf{x}_{0}=\mathbf{0}$, the corresponding integral curve is a one parameter subgroup of $G$, and that conversely, any one parameter subgroup of $G$ represents the integral curve of a right invariant field on $G$, through $\mathbf{0}$. (See the text above (9) for the definition of a one parameter subgroup).

Define

$$
\boldsymbol{\ell}_{a}(\mathbf{x})=\nabla_{1} \boldsymbol{\psi}(\mathbf{0}, \mathbf{x}) \mathbf{e}_{a}, \quad a=1,2,3 .
$$

Then one can show, as in Parry[4], that these fields are right invariant, and that an arbitrary right invariant field $\boldsymbol{\nu}(\cdot)$ can be written in the form

$$
\boldsymbol{\nu}(\mathbf{x})=\left(\mathbf{f}_{a} \cdot \boldsymbol{\nu}(\mathbf{0})\right) \boldsymbol{\ell}_{a}(\mathbf{x}),
$$

where $\mathbf{f}_{1}, \mathbf{f}_{2}, \mathbf{f}_{3}$ is dual to the basis $\left\{\mathbf{e}_{1}, \mathbf{e}_{2}, \mathbf{e}_{3}\right\}$. From(20), the fields $\boldsymbol{\ell}_{1}(\cdot), \boldsymbol{\ell}_{2}(\cdot), \boldsymbol{\ell}_{3}(\cdot)$ defined by (19) provide a basis for the vector space of all right invariant fields on $G$. Also from $(20)$, it follows that the vector field $\boldsymbol{\nu}(\cdot)$ on $G$ is specified once $\boldsymbol{\nu}(\mathbf{0})$ is prescribed.

Now (18) has a solution defined for all $t \in \mathbb{R}$, and thereby defines a mapping $\exp (t \boldsymbol{\nu}): G \rightarrow G$ given by

$$
\exp (t \boldsymbol{\nu})\left(\mathbf{x}_{0}\right)=\mathbf{x}(t)
$$

where one understands the notation $\exp (t \boldsymbol{\nu})$ as follows: let $\boldsymbol{\nu}(\cdot)$ be the unique right invariant vector field on $G$ such that $\boldsymbol{\nu}(\mathbf{0})=\boldsymbol{\nu}$, then $\exp (t \boldsymbol{\nu})$ is the mapping that sends $\mathbf{x}(0) \equiv \mathbf{x}_{0}$ to $\mathbf{x}(t)$ via (18), noting (18) implies that if $t \boldsymbol{\nu}=t^{\prime} \boldsymbol{\nu}^{\prime}$, where $t \neq t^{\prime}, \boldsymbol{\nu} \neq \boldsymbol{\nu}^{\prime}$, then $\exp (t \boldsymbol{\nu})=\exp \left(t^{\prime} \boldsymbol{\nu}^{\prime}\right)$.

Also define the group element $e^{(t \boldsymbol{\nu})} \in G$ (as opposed to the mapping $\exp (t \boldsymbol{\nu})$ ) by

$$
e^{(t \boldsymbol{\nu})}=\exp (t \boldsymbol{\nu})(\mathbf{0}) .
$$

Note that $e^{()}: \mathbb{R}^{3} \rightarrow G \cdot e^{()}$is called the exponential mapping of the Lie algebra (here $\mathbb{R}^{3}$ ) to the Lie group. It is a standard result that

$$
\exp (t \boldsymbol{\nu})(\mathbf{x})=\boldsymbol{\psi}\left(e^{(t \boldsymbol{\nu})}, \mathbf{x}\right) \equiv e^{(t \boldsymbol{\nu})} \mathbf{x}, \quad t \boldsymbol{\nu} \in \mathbb{R}^{3}, \mathbf{x} \in G .
$$


2.2 Group and algebra homomorphisms

Let $\mathfrak{g}$ and $\mathfrak{h}$ be Lie algebras with Lie brackets $[\cdot, \cdot]_{\mathfrak{g}},[\cdot, \cdot]_{\mathfrak{h}}$ respectively. (In the context of this paper, both brackets $[\cdot, \cdot]_{\mathfrak{g}},[\cdot, \cdot]_{\mathfrak{h}}$ map $\left.\mathbb{R}^{3} \times \mathbb{R}^{3} \rightarrow \mathbb{R}^{3}\right)$. A Lie algebra homomorphism is a linear transformation $L: \mathfrak{g} \rightarrow \mathfrak{h}$ which satisfies

$$
[L \mathbf{x}, L \mathbf{y}]_{\mathfrak{h}}=L[\mathbf{x}, \mathbf{y}]_{\mathfrak{g}}, \quad \mathbf{x}, \mathbf{y} \in \mathfrak{g}
$$

If $C_{i j k}^{\mathfrak{g}}, C_{i j k}^{\mathfrak{h}}$ are the structure constants for $\mathfrak{g}, \mathfrak{h}$ respectively, then (13),(16),(24) imply

$$
C_{i j k}^{\mathfrak{h}} L_{j p} L_{k q}=L_{i r} C_{r p q}^{\mathfrak{g}}
$$

where $L \mathbf{e}_{i}=L_{j i} \mathbf{e}_{j}, i, j=1,2,3$.

Let $G$ and $H$ be Lie groups with group multiplication functions $\psi_{G}, \psi_{H}$ respectively. A smooth mapping $\phi: G \rightarrow H$ is a Lie group homomorphism if

$$
\psi_{H}(\phi(\mathbf{x}), \phi(\mathbf{y}))=\phi\left(\psi_{G}(\mathbf{x}, \mathbf{y})\right), \quad \mathbf{x}, \mathbf{y} \in G .
$$

Then if $\mathfrak{g}$ is the Lie algebra of $G$, and $\mathfrak{h}$ is the Lie algebra of $H$, and $\phi: G \rightarrow H$ is a Lie group homomorphism, it is a fact that $\nabla \phi(\mathbf{0}) \equiv L$ is a Lie algebra homomorphism. Conversely if $L$ satisfies (24), then there exists a Lie group homomorphism $\phi$ such that $\nabla \phi(\mathbf{0})=L$. Also,

$$
\phi\left(e^{\boldsymbol{\nu}}\right)=e^{(\nabla \phi(\mathbf{0}) \boldsymbol{\nu})}, \quad \boldsymbol{\nu} \in \mathfrak{g} \equiv \mathbb{R}^{3},
$$

where $\phi$ satisfies (26), where the exponential on the left hand side of (27) is the exponential which maps $\mathfrak{g}$ to $G$, and that on the right hand side maps $\mathfrak{h}$ to $H$. Relation (27) allows one to calculate the Lie group homomorphisms explicitly if the Lie algebra homomorphisms are found by solving (25).

\subsection{Nilpotent groups and algebras}

Let $G$ be a three dimensional Lie group, with commutator $(\mathbf{x}, \mathbf{y}) \equiv \mathbf{x}^{-1} \mathbf{y}^{-1} \mathbf{x y}$. Let $G \equiv$ $G_{0}$ and define $G_{1} \equiv\left(G, G_{0}\right)$, the group generated by elements of the form $(\mathbf{x}, \mathbf{y}), \mathbf{x} \in$ $G, \mathbf{y} \in G_{0}$. Define $G_{k} \equiv\left(G, G_{k-1}\right)$ inductively, $k \geq 1$. $G$ is called nilpotent if and only if $G_{k}$ is the trivial group $\{\mathbf{0}\}$ for sufficiently large $k$. For three dimensional nilpotent groups, $G \equiv G_{0} \supseteq G_{1} \supseteq G_{2}=\{\mathbf{e}\}$, where $\mathbf{e}$ is a temporary notation for the group identity $\mathbf{0}$.

Let $\mathfrak{g}$ be the Lie algebra corresponding to a Lie group $G$, with Lie bracket $[\mathbf{x}, \mathbf{y}], \mathbf{x}, \mathbf{y} \in$ $\mathfrak{g}$. Let $\mathfrak{g} \equiv \mathfrak{g}_{0}$ and define $\mathfrak{g}_{1} \equiv\left[\mathfrak{g}, \mathfrak{g}_{0}\right]$, the subspace generated by elements of the form $[\mathbf{x}, \mathbf{y}], \mathbf{x} \in \mathfrak{g}, \mathbf{y} \in \mathfrak{g}_{0}$. Define $\mathfrak{g}_{k} \equiv\left[\mathfrak{g}, \mathfrak{g}_{k-1}\right]$ inductively, $k \geq 1$. $\mathfrak{g}$ is called nilpotent if and only if $\mathfrak{g}_{k}$ is the trivial subspace $\{\mathbf{0}\}$ for sufficiently large $k$. For three dimensional nilpotent algebras, $\mathfrak{g} \equiv \mathfrak{g}_{0} \supseteq \mathfrak{g}_{1} \supseteq \mathfrak{g}_{2}=\{\mathbf{0}\}$.

A Lie group is nilpotent if and only if the corresponding Lie algebra is nilpotent (Gorbatsevich, Onishchik, Vinberg [10]).

Note the following identities, valid for any three elements $\mathbf{a}, \mathbf{b}, \mathbf{c}$ of a group $G$, due to Hall[11] (cf. Magnus, Karrass, Solitar [12])

$$
(\mathbf{a}, \mathbf{b})(\mathbf{b}, \mathbf{a})=\mathbf{e},(\mathbf{a}, \mathbf{b c})=(\mathbf{a}, \mathbf{c})(\mathbf{a}, \mathbf{b})((\mathbf{a}, \mathbf{b}), \mathbf{c}),(\mathbf{a b}, \mathbf{c})=(\mathbf{a}, \mathbf{c})((\mathbf{a}, \mathbf{c}), \mathbf{b})(\mathbf{b}, \mathbf{c}) .
$$


In the case of three dimensional nilpotent Lie Groups $G,((\mathbf{a}, \mathbf{b}), \mathbf{c})=\mathbf{e}$ for all $\mathbf{a}, \mathbf{b}, \mathbf{c} \in$ $G$, so (28) gives

$$
(\mathbf{a}, \mathbf{b})(\mathbf{b}, \mathbf{a})=\mathbf{e},(\mathbf{a}, \mathbf{b c})=(\mathbf{a}, \mathbf{c})(\mathbf{a}, \mathbf{b}),(\mathbf{a b}, \mathbf{c})=(\mathbf{a}, \mathbf{c})(\mathbf{b}, \mathbf{c})
$$

For example, let $G^{\prime}$ be the three dimensional nilpotent Lie group with composition function

$$
\boldsymbol{\psi}(\mathbf{x}, \mathbf{y})=\mathbf{x}+\mathbf{y}+x_{1} y_{2} \mathbf{e}_{3}, \quad \mathbf{x}, \mathbf{y} \in \mathbb{R}^{3}
$$

Then

$$
\frac{\partial^{2} \psi_{i}}{\partial x_{j} \partial y_{k}} \equiv \delta_{i 3} \delta_{j 1} \delta_{k 2}, \quad C_{i j k}=\left(\delta_{j 1} \delta_{k 2}-\delta_{k 1} \delta_{j 2}\right) \delta_{i 3}=\delta_{i 3} \varepsilon_{3 j k},
$$

where $\delta_{i j}, \varepsilon_{i j k}$ are the Kronecker delta and permutation symbol, respectively. Hence

$$
[\mathbf{x}, \mathbf{y}]=\mathbf{e}_{3}\left(\mathbf{e}_{3} \cdot \mathbf{x} \wedge \mathbf{y}\right),\left[\mathbf{x}, \mathbf{e}_{3}\right]=\mathbf{0}
$$

so the Lie algebra, and hence the Lie group, is nilpotent, $\mathfrak{g}_{2}=\{\mathbf{0}\}$. As a matter of interest, note that if one introduces the maps $\mathbf{r}: \mathbb{R}^{3} \rightarrow M^{3 \times 3}$ by

$$
\mathbf{r}(\mathbf{x})=\mathbf{r}\left(x_{1}, x_{2}, x_{3}\right)=\left(\begin{array}{ccc}
1 & x_{1} & x_{3} \\
0 & 1 & x_{2} \\
0 & 0 & 1
\end{array}\right),
$$

where $\mathbf{x}=x_{i} \mathbf{e}_{i}$, then

$$
\mathbf{r}(\mathbf{x}) \mathbf{r}(\mathbf{y})=\left(\begin{array}{ccc}
1 & x_{1}+y_{1} & x_{3}+y_{3}+x_{1} y_{2} \\
0 & 1 & x_{2}+y_{2} \\
0 & 0 & 1
\end{array}\right),
$$

where the left hand side of (34) represents matrix multiplication of $\mathbf{r}(\mathbf{x})$ and $\mathbf{r}(\mathbf{y})$. Thus

$$
\mathbf{r}(\mathbf{x}) \mathbf{r}(\mathbf{y})=\mathbf{r}(\boldsymbol{\psi}(\mathbf{x}, \mathbf{y}))
$$

so that group multiplication (30) is converted to matrix multiplication via (33).

One calculates further from (30) that $\boldsymbol{\ell}_{a}(\mathbf{x})=\nabla_{1} \boldsymbol{\psi}(\mathbf{0}, \mathbf{x}) \mathbf{e}_{a}, a=1,2,3$, gives

$$
\ell_{1}(\mathbf{x})=\mathbf{e}_{1}+x_{2} \mathbf{e}_{3}, \ell_{2}(\mathbf{x})=\mathbf{e}_{2}, \ell_{3}(\mathbf{x})=\mathbf{e}_{3} .
$$

Also, if $\dot{\mathbf{x}}=\boldsymbol{\nu}(\mathbf{x}(t))=\nu_{a} \boldsymbol{\ell}_{a}(\mathbf{x}(t))$, from (18) and (20), where $\nu_{a} \equiv \mathbf{f}_{a} \cdot \boldsymbol{\nu}(\mathbf{0}), \boldsymbol{\nu}(\mathbf{0})=$ $\nu_{a} \mathbf{e}_{a}$, one finds

$$
\exp (\boldsymbol{\nu} t)(\mathbf{x})=\mathbf{x}+\boldsymbol{\nu} t+\left(\frac{1}{2}\left(\nu_{1} t\right)\left(\nu_{2} t\right)+\left(\nu_{1} t\right) x_{2}\right) \mathbf{e}_{3}, \quad e^{(\boldsymbol{\nu} t)}=\boldsymbol{\nu} t+\frac{1}{2}\left(\nu_{1} t\right)\left(\nu_{2} t\right) \mathbf{e}_{3},
$$

and one may check that $\boldsymbol{\psi}\left(e^{(\boldsymbol{\nu} t)}, \mathbf{x}\right)=\exp (\boldsymbol{\nu} t)(\mathbf{x})$.

It is generally true that if $G$ is nilpotent and $\mathfrak{g}$ is the corresponding Lie algebra, that the exponential mapping from $\mathfrak{g}$ to $G$ is a homeomorphism (i.e. the exponential is a continuous bijection, and so is its inverse). 
2.4 The canonical group $J$, the Campbell-Baker-Hausdorff formula

According to Varadarajan [13], there is a one-to-one correspondence between isomorphism classes of Lie algebra and isomorphism classes of Lie groups. ( $\phi: G \rightarrow H$ is an isomorphism of Lie groups $G, H$ if (26) holds, $\phi^{-1}$ exists and is also smooth, etc..)

Let $\boldsymbol{\psi}$ be the composition function for a three dimensional Lie Group $G$, and let $e^{(\cdot)}: \mathfrak{g} \equiv \mathbb{R}^{3} \rightarrow G$ be the exponential function. The Campbell-Baker-Hausdorff (CBH) formula gives an explicit expression for the quantity $\mathbf{c}$ in the relation,

$$
e^{(\mathbf{c})}=e^{(\mathbf{a})} e^{(\mathbf{b})}, \quad a, b \in \mathbb{R}^{3} .
$$

One finds the full formula in Varadarajan[13]. I give here only the formula as it applies to three dimensional nilpotent Lie groups $G$, it is

$$
\mathbf{c}=\mathbf{a}+\mathbf{b}+\frac{1}{2}[\mathbf{a}, \mathbf{b}]
$$

The simple form of (39) exposes a remarkable fact - the expression on the right hand side of (39) depends only on the Lie bracket $[\cdot, \cdot]$, i.e. it only depends on the Lie algebra $\mathfrak{g}$, it does not depend on the choice of group $G$ in the isomorphism class of groups which have the Lie algebra determined by the given bracket operation.

Now put

$$
\mathbf{c}=\psi^{\prime}(\mathbf{a}, \mathbf{b})
$$

and note that $\boldsymbol{\psi}^{\prime}$ satisfies (4) by virtue of $[[\mathbf{a}, \mathbf{b}], \mathbf{c}]=\mathbf{0}$, (5) by (39), (6) if one puts $\mathbf{a}^{-1}=-\mathbf{a}$. So the CBH formula (39) delivers a function $\boldsymbol{\psi}^{\prime}$, via (40), which one can regard as a group composition function on the vector space associated with the given Lie algebra $\left(\mathbb{R}^{3}\right.$ in this case). I call this group the canonical group $J$ associated with the given structure constants (i.e. with the given Lie algebra).

When (1) holds, it turns out (see the first sentence of section 5) that the structure constants have the form

$$
C_{i j k}=\lambda \varepsilon_{r j k} p_{i} p_{r}
$$

where the parameters $\lambda, \mathbf{p}$ that occur in (41) are not necessarily the same as the parameters denoted by those letters in (1). Then, the composition function in the group $J$ (dropping the prime in $\psi^{\prime}$ ) is given by

$$
\psi(\mathbf{x}, \mathbf{y})=\mathbf{x}+\mathbf{y}+\frac{1}{2} \lambda \mathbf{p}(\mathbf{p} \cdot \mathbf{x} \wedge \mathbf{y})
$$

and one calculates that $\boldsymbol{\ell}_{a}(\mathbf{x})=\nabla_{1} \boldsymbol{\psi}(\mathbf{0}, \mathbf{x}) \mathbf{e}_{a}=\mathbf{e}_{a}+\frac{1}{2} \lambda \mathbf{p}\left(\mathbf{x} \wedge \mathbf{p} \cdot \mathbf{e}_{a}\right)$, and that from $\dot{\mathbf{x}}=\nu_{a} \ell_{a}(\mathbf{x})=\boldsymbol{\nu}+\frac{1}{2} \lambda \mathbf{p}(\mathbf{x} \wedge \mathbf{p} \cdot \boldsymbol{\nu})$, one obtains

$$
\exp (\boldsymbol{\nu} t)(\mathbf{x})=\mathbf{x}+\boldsymbol{\nu} t+\frac{1}{2} \lambda \mathbf{p}(\mathbf{x} \wedge \mathbf{p} \cdot \boldsymbol{\nu} t)
$$

Therefore, $\exp (\boldsymbol{\nu} t)(\mathbf{0})=\boldsymbol{\nu} t$, which implies that the one parameter groups in $J$ are straight lines, and that the corresponding exponential mapping is

$$
e^{(\mathbf{x})}=\exp (\mathbf{x})(\mathbf{0})=\mathbf{x}, \quad \mathbf{x} \in \mathbb{R}^{3} .
$$

That is, Lie group and Lie algebra elements may be identified, in $J$, via (44). One calculates that the commutator and the Lie bracket coincide, with this identification. 
Now by a slight extension of (27), if a linear transformation $L$ is a Lie algebra automorphism (i.e. an isomorphism from the algebra to itself), there is a Lie group automorphism $\phi: J \rightarrow J$ such that $\nabla \phi(\mathbf{0})=L$. Then $\phi\left(\mathbf{e}^{(\mathbf{x})}\right)=e^{(\nabla \boldsymbol{\phi}(0) \mathbf{x})}$ gives, noting that the exponentials on both sides of this relation satisfy (44), that

$$
\phi(\mathbf{x})=\nabla \phi(\mathbf{0}) \mathbf{x}, \quad \mathbf{x} \in \mathfrak{g} \equiv J .
$$

Relation (45) shows that the automorphisms of $J$ are linear mappings ('homogeneous deformations' in continuum mechanical terms), and this fact helps a great deal when one comes to calculate the symmetries of discrete subgroups of $J$ later on.

\section{Elastic deformations are Lie group isomorphisms}

Let $\ell_{1}(\cdot), \ell_{2}(\cdot), \ell_{3}(\cdot)$ be smooth linearly independent 'lattice' vector fields defined on a region $B \subseteq \mathbb{R}^{3}$. Let $\mathbf{u}: B \rightarrow B^{\prime}$ be an invertible smooth map with $\mathbf{u}(\mathbf{0})=\mathbf{0}$, for simplicity. If the lattice vector fields are transformed by the map $\mathbf{u}$ to fields $\ell_{1}^{\prime}(\cdot), \ell_{2}^{\prime}(\cdot), \ell_{3}^{\prime}(\cdot)$ such that

$$
\ell_{a}^{\prime}(\mathbf{u}(\mathbf{x}))=\nabla \mathbf{u}(\mathbf{x}) \ell_{a}(\mathbf{x}), \quad \mathbf{x} \in B
$$

one says that the fields $\boldsymbol{\ell}_{a}^{\prime}(\cdot), a=1,2,3$ are related to the fields $\boldsymbol{\ell}_{a}(\cdot)$ by elastic deformation.

Suppose that the fields $\ell_{1}(\cdot), \ell_{2}(\cdot), \ell_{3}(\cdot)$ are such that $S$, calculated via $(2)$, is constant. Then, since $S$ is an elastic invariant, it follows if one calculates $S^{\prime}$ for the fields $\ell_{1}^{\prime}(\cdot), \ell_{2}^{\prime}(\cdot), \ell_{3}^{\prime}(\cdot)$ via the analogue of $(2)$, then $S^{\prime} \equiv S$ is also a constant. By virtue of the remarks surrounding (3), it follows that the two partial differential systems

$$
\boldsymbol{\ell}_{a}(\boldsymbol{\psi}(\mathbf{x}, \mathbf{y}))=\nabla_{1} \boldsymbol{\psi}(\mathbf{x}, \mathbf{y}) \boldsymbol{\ell}_{a}(\mathbf{x}), \boldsymbol{\ell}_{a}^{\prime}\left(\boldsymbol{\psi}^{\prime}\left(\mathbf{x}^{\prime}, \mathbf{y}^{\prime}\right)\right)=\nabla_{1} \psi^{\prime}\left(\mathbf{x}^{\prime}, \mathbf{y}^{\prime}\right) \ell_{a}^{\prime}\left(\mathbf{x}^{\prime}\right), \quad a=1,2,3,
$$

have solutions for the functions $\psi, \psi^{\prime}$ defined on $B \times B, B^{\prime} \times B^{\prime}$ respectively. The functions $\boldsymbol{\psi}, \boldsymbol{\psi}^{\prime}$ have the properties required to be Lie group composition functions let us denote $B$ by $G, B^{\prime}$ by $G^{\prime}$, in recognition of this, and admit that (47) implies that the vector fields $\ell_{a}(\cdot), \ell_{a}^{\prime}(\cdot)$ may be interpreted as right invariant fields on the groups $G, G^{\prime}$ respectively. Then $\mathbf{u}: G \rightarrow G^{\prime}$ and one can check that if (46) and the first of (47) hold, and one defines $\psi^{\prime}$ by

$$
\psi^{\prime}(\mathbf{u}(\mathbf{x}), \mathbf{u}(\mathbf{y}))=\mathbf{u}(\boldsymbol{\psi}(\mathbf{x}, \mathbf{y})),
$$

then the second of (47) also holds. Thus, by comparing (26) and (48), noting that $\mathbf{u}^{-1}$ exists and is smooth, one sees that elastic deformations of lattice vector fields which have constant ddt provide Lie group isomorphisms of Lie groups defined as above. Furthermore, if one denotes the Lie algebras of $G, G^{\prime}$ by $\mathfrak{g}, \mathfrak{g}^{\prime}$ respectively, then $\nabla \mathbf{u}(\mathbf{0})$ is a Lie algebra isomorphism which preserves the structure constants of $\mathfrak{g}, \mathfrak{g}^{\prime}$ in the sense that (24) holds with $L=\nabla \mathbf{u}(\mathbf{0}), \mathfrak{h} \equiv \mathfrak{g}^{\prime}$.

According to Elzanowski and Parry [3], the connection between the ddt $S$, defined by (2), and the structure constants (defined via (14)) of the group $G$ which has right invariant fields $\ell_{1}(\cdot), \ell_{2}(\cdot), \ell_{3}(\cdot)$, is

$$
C_{i j k} \ell_{r j}(\mathbf{0}) \ell_{s k}(\mathbf{0})=\varepsilon_{p r s} S_{k p} \ell_{k i}(\mathbf{0}) .
$$

Recall that the structure constants $C_{i j k}$ are defined via (13) and (16), so that $[\mathbf{x}, \mathbf{y}]=C_{i j k} x_{j} y_{k} \mathbf{e}_{i}$, where $\mathbf{x}=x_{i} \mathbf{e}_{i}$, etc.. If one defines structure constants $C_{i j k}^{\prime}$ 
relative to a different basis $\mathbf{e}_{1}^{\prime}, \mathbf{e}_{2}^{\prime}, \mathbf{e}_{3}^{\prime}$, where $\mathbf{e}_{i}^{\prime}=\xi_{i j} \mathbf{e}_{j}, i=1,2,3$, say, then it follows that

$$
C_{r p q}^{\prime} \xi_{r i}=C_{i j k} \xi_{p j} \xi_{q k}
$$

Now let $C_{i j k}^{*}$ be the structure constants with respect to the basis $\boldsymbol{\ell}_{1}(\mathbf{0}), \boldsymbol{\ell}_{2}(\mathbf{0}), \boldsymbol{\ell}_{3}(\mathbf{0})$, where $\boldsymbol{\ell}_{i}(\mathbf{0})=\ell_{i j}(\mathbf{0}) \mathbf{e}_{j}, i=1,2,3$. Then from (50)

$$
C_{r p q}^{*} \ell_{r i}(\mathbf{0})=C_{i j k} \ell_{p j}(\mathbf{0}) \ell_{q k}(\mathbf{0})
$$

So from (49)

$$
C_{r p q}^{*}=\varepsilon_{\ell p q} S_{r \ell}
$$

Thus the dislocation density tensor is simply related to the structure constants with respect to the basis $\boldsymbol{\ell}_{1}(\mathbf{0}), \ell_{2}(\mathbf{0}), \ell_{3}(\mathbf{0})$. In particular, the components of $S$ are rational if and only if the components $C_{r p q}^{*}$ are rational, and this is a fact which will be required later.

Note that the structure constant depend just on the composition function in $G$, whereas from (49) the ddt $S$ depends also on the choice of right invariant fields (in fact on the values of these fields at $\mathbf{0}$ ). From (46) and the simplifying assumption $\mathbf{u}(\mathbf{0})=\mathbf{0}$,

$$
\ell_{a}^{\prime}(\mathbf{0})=\nabla \mathbf{u}(\mathbf{0}) \boldsymbol{\ell}_{a}(\mathbf{0}) \equiv L \boldsymbol{\ell}_{a}(\mathbf{0}), \quad a=1,2,3
$$

One may check via (53), that (49) and its analogue for $G^{\prime}$ are consistent with the fact that $S$ is an elastic invariant. Also, when $S$ has the form (1), one may check that (49) implies that the structure constants necessarily have the form that was employed in (41).

For example, let $G^{\prime}$ be as defined in 2.3, with composition function (30), and let $J^{\prime}$ denote that particular canonical group which has the same structure constants as $G^{\prime}$, so that from (42)

$$
\psi(\mathbf{x}, \mathbf{y})=\mathbf{x}+\mathbf{y}+\frac{1}{2}\left(\mathbf{e}_{3} \cdot \mathbf{x} \wedge \mathbf{y}\right) \mathbf{e}_{3}, \quad \mathbf{x}, \mathbf{y} \in J^{\prime}
$$

The structure constants in $G^{\prime}$, and $J^{\prime}$, are $C_{i j k}=\delta_{3 i} \varepsilon_{3 j k}$, so the Lie algebra homomorphisms $L: \mathfrak{g}^{\prime} \rightarrow \mathbf{j}^{\prime}$ (in the obvious notation) satisfy

$$
L_{i 3} \varepsilon_{3 p q}=\delta_{i 3} \varepsilon_{3 j k} L_{j p} L_{k q},
$$

via (25). It follows that

$$
L_{13}=L_{23}=0, \quad L_{33}=L_{11} L_{22}-L_{12} L_{21} .
$$

If $L$ is to be an isomorphism, then it must be invertible, and so from the second of (56),

$$
L_{33} \neq 0
$$

Note also that the Lie group automorphisms of $J^{\prime}$ (i.e. the isomorphisms of $J^{\prime}$ to $J^{\prime}$, or the 'symmetries' of $J^{\prime}$ ) have the same form, via (45). 
4 Mal'cev's coordinates, canonical bases for discrete groups

The purpose of this paper is to discuss symmetries of discrete sets of points associated with a defective crystal that has constant ddt. The sets of points that are considered turn out to be discrete subgroups of Lie groups, and the symmetries of those subgroups are elastic deformations that preserve the ddt - they are automorphisms of the subgroups. In this section I first outline the way that these subgroups were introduced in Cermelli and Parry [2], Parry [4], [5], next I paraphrase Mal'cev's perspective and remark that the two positions coincide in the context of this paper, finally I outline Mal'cev's results, so far as they relate to the topics at hand.

In [2], [4], [5], the subgroups arise in the following way: choose three smooth right invariant fields $\ell_{1}(\cdot), \ell_{2}(\cdot), \ell_{3}(\cdot)$ to specify the texture of a crystal with constant ddt $S$, as in Davini's prescription. Let the corresponding Lie groups be denoted $G$. From (49) calculate the structure constants $C_{i j k}$ of a corresponding Lie algebra. There is an isomorphism (elastic deformation) from $G$ to the canonical group $J$ that has the same structure constants, denote it by $\boldsymbol{\theta}, \boldsymbol{\theta}: G \rightarrow J$, and let $\boldsymbol{\ell}_{a}^{\prime}(\boldsymbol{\theta}(\mathbf{x}))=\nabla \boldsymbol{\theta}(\mathbf{x}) \boldsymbol{\ell}_{a}(\mathbf{x})$, $i=1,2,3$, by analogy with (46). It follows that attention may be restricted to discrete subgroups of $J$, without loss of generality, for if $D^{\prime}$ is a discrete subgroup of $G$, then $\boldsymbol{\theta}\left(D^{\prime}\right)$ is a discrete subgroup of $J$.

Suppose now that $\boldsymbol{\ell}_{a}(\cdot), a=1,2,3$ are right invariant fields defined on $J$. Let $\tilde{\mathbf{x}} \in J$ and say that $\tilde{\mathbf{y}} \in J$ is a neighbour of $\tilde{\mathbf{x}}$ if and only if there exists an index $a \in\{1,2,3\}$ such that

either $\frac{d \mathbf{x}}{d t}(t)=\ell_{a}(\mathbf{x}(t)), \mathbf{x}(0)=\tilde{\mathbf{x}}, \mathbf{x}(1)=\tilde{\mathbf{y}}$, or $\frac{d \mathbf{x}}{d t}(t)=\ell_{a}(\mathbf{x}(t)), \mathbf{x}(0)=\tilde{\mathbf{y}}, \mathbf{x}(1)=\tilde{\mathbf{x}}$.

Thus $\tilde{\mathbf{x}}$ and $\tilde{\mathbf{y}}$ are neighbours of each other if and only if the 'unit' flow along some lattice vector field maps $\tilde{\mathbf{x}}$ to $\tilde{\mathbf{y}}$ or vice versa. (This is a generalization of the 'nearest neighbour' idea for a cubic lattice). Let $D \subset J$ be a set such that $\mathbf{0} \in D$ and such that if $\mathbf{x} \in D$, then the neighbours of $\mathbf{x}$ are elements of $D$. Then since $\mathbf{0} \in D$, from (22) and (23) one obtains $e^{\left(\boldsymbol{\ell}_{i}\right)} \in D, e^{-\left(\boldsymbol{\ell}_{i}\right)} \in D$, where $\boldsymbol{\ell}_{i} \equiv \boldsymbol{\ell}_{i}(\mathbf{0}), i=1,2,3$ (noting that $\left.\left(e^{\left(\ell_{i}\right)}\right)^{-1}=e^{-\left(\ell_{i}\right)}\right)$. Also if $\mathbf{x} \in D$, then $\boldsymbol{\alpha} \mathbf{x} \in D$, where $\boldsymbol{\alpha}$ is any of the six elements $e^{\left(\boldsymbol{\ell}_{i}\right)}, e^{-\left(\boldsymbol{\ell}_{i}\right)}, i=1,2,3$. So $D$ includes all elements of $J$ which have the form

$$
\mathbf{x}=\boldsymbol{\alpha}_{1} \boldsymbol{\alpha}_{2} \ldots \boldsymbol{\alpha}_{n}
$$

where $n$ is arbitrary, and each $\boldsymbol{\alpha}_{i}, i=1,2, \ldots n$ is one of $e^{\left(\boldsymbol{\ell}_{a}\right)}, e^{-\left(\boldsymbol{\ell}_{a}\right)}, a=1,2,3$. Suppose that $D$ has no other elements. Then $D$ is a subgroup of $J$ (with group operation corresponding to juxtaposition of expressions such as that on the right hand side of (59), recognizing that $e^{\left(\ell_{a}\right)} e^{-\left(\ell_{a}\right)}$ is the group identity). Cermelli and Parry [2], Parry [4] showed that $D$ is a discrete subgroup of $J$ if the ddt is rational (i.e. each $S_{a b}$ is rational, $a, b=1,2,3)$ - the assumption that $S$ is rational is adopted henceforward. $D$ is said to be generated by the three elements $e^{\left(\boldsymbol{\ell}_{1}\right)}, e^{\left(\boldsymbol{\ell}_{2}\right)}, e^{\left(\boldsymbol{\ell}_{3}\right)}$, when $(59)$ holds for all $\mathbf{x} \in D$.

Note that, generally, an element of $D$ has many representations of the form (59). One may think of the representation $\mathbf{x}=\boldsymbol{\alpha}_{1} \boldsymbol{\alpha}_{2} \ldots \boldsymbol{\alpha}_{n} \in D$ as specifying a 'path' from the origin (in $D$ ) to the point $\mathbf{x}$, via the intermediate points $\boldsymbol{\alpha}_{n}, \boldsymbol{\alpha}_{n-1} \boldsymbol{\alpha}_{n}, \ldots$, $\boldsymbol{\alpha}_{2} \ldots \boldsymbol{\alpha}_{n-1} \boldsymbol{\alpha}_{n}$. Many paths lead to the same point $\mathbf{x}$, in general (think of a perfect lattice, for example), so suppose

$$
\mathbf{x}=\boldsymbol{\alpha}_{1} \boldsymbol{\alpha}_{2} \ldots \boldsymbol{\alpha}_{n}=\boldsymbol{\beta}_{1} \boldsymbol{\beta}_{2} \ldots \boldsymbol{\beta}_{m}
$$


where each of $\boldsymbol{\alpha}_{i}, i=1,2 \ldots n ; \boldsymbol{\beta}_{j}, j=1,2 \ldots m$, is one of the generators or the inverse of one of the generators. Thus

$$
\boldsymbol{\alpha}_{1} \boldsymbol{\alpha}_{2} \ldots \boldsymbol{\alpha}_{n} \boldsymbol{\beta}_{m}^{-1} \boldsymbol{\beta}_{m-1}^{-1} \ldots \boldsymbol{\beta}_{1}^{-1}=\mathbf{0}
$$

and one sees that the non-uniqueness of the representation (59) corresponds precisely to the existence of non trivial relations (such as (61)) between the generators and their inverses. Said differently, this non-uniqueness corresponds to the existence of 'circuits' in $D$ - indeed one can think of the elements of $D$ as the set of paths in $D$, modulo the set of circuits in $D$. (This is the standpoint in texts on combinatorial group theory, such as Magnus, Karrass, Solitar [12], Johnson [14], where the elements of $D$ are represented as elements of the free group on the generators of $D$, modulo the normal closure of the relators).

The above construction, adopted in Cermelli and Parry [2], Parry [4], [5], places the analogy with the construction of a perfect lattice (in the case $S=0$ ) or its heart. Mal'cev [1], on the other hand, considers discrete subgroups $D$ of a general Lie group $G$, a priori, without assuming that $D$ has a finite number of generators. Nominally, then, his position is more general than that adopted in [2], [4], [5]. However, he finds it useful to restrict attention to uniform discrete subgroups of $G$ : a discrete subgroup of $G$ is uniform if the left coset space $G / D$ is compact - this is the generalization of the requirement, in the case $S=0$, that $\mathbb{R}^{3} / L$ (which is the 'unit cell' of the lattice, with appropriate identification of boundary points) is compact. Then, he discovers that this criterion (that the subgroup be uniform), and the restriction to three dimensional nilpotent Lie groups, together imply that $D$ is generated by three elements. He also shows: in order that $G$ contains a uniform discrete subgroup $D$, it is necessary and sufficient that the corresponding Lie algebra $\mathfrak{g}$ have rational structure constants with respect to an appropriate basis. Bearing equation (52) in mind, one sees that the two perspectives coincide, in the context of this paper. So I paraphrase Mal'cev's results below, as they particularize to the three dimensional case.

\subsection{Mal'cev's coordinates}

Let $G$ be a connected and simply connected three dimensional nilpotent Lie group, let $\mathfrak{g}$ be the corresponding Lie algebra, and let $J$ be the corresponding canonical group. Recall that a subspace $\mathbf{h} \subseteq \mathfrak{g}$ is an ideal if and only if $[\mathbf{h}, \mathfrak{g}] \subseteq \mathbf{h}$.

Select in $\mathfrak{g}$ an ordered basis $\left\{\mathbf{g}_{1}, \mathbf{g}_{2}, \mathbf{g}_{3}\right\}$ such that

$-\left\{a_{2} \mathbf{g}_{2}+a_{3} \mathbf{g}_{3} ; a_{2}, a_{3} \in \mathbb{R}\right\} \equiv \mathfrak{g}_{2}$ is an ideal in $\mathfrak{g}$,

$-\left\{a_{3} \mathbf{g}_{3} ; a_{3} \in \mathbb{R}\right\} \equiv \mathfrak{g}_{3}$ is an ideal in $\mathfrak{g}$.

Each element of $\mathfrak{g}$, and therefore each element of $J$, can be uniquely represented in the form

$$
\mathbf{g}=a_{1} \mathbf{g}_{1}+a_{2} \mathbf{g}_{2}+a_{3} \mathbf{g}_{3}
$$

Then by definition

- the numbers $a_{1}, a_{2}, a_{3}$ are the 'coordinates of the first kind' of $\mathbf{g}$,

- the vectors $\mathbf{g}_{1}, \mathbf{g}_{2}, \mathbf{g}_{3}$ are the corresponding 'system of coordinates of the first kind'. 
For example, if $[\mathbf{x}, \mathbf{y}]=\lambda \mathbf{p}(\mathbf{p} \cdot \mathbf{x} \wedge \mathbf{y}), \lambda \in \mathbb{R}$ and $\{\boldsymbol{\ell}, \mathbf{m}, \mathbf{p}\}$ is a basis of $\mathbb{R}^{3}$, the ordered basis $\{\boldsymbol{\ell}, \mathbf{m}, \mathbf{p}\}$ is a system of coordinates of the first kind (because $\mathfrak{g}_{3} \equiv \mathbb{R} \mathbf{p},[\mathbb{R} \mathbf{p}, \mathbf{y}]=$ $\mathbf{0}, \mathbf{y} \in \mathfrak{g}$, and $\left.\left[\mathfrak{g}_{2}, \mathbf{y}\right]=\mathbb{R} \mathbf{p} \subseteq \mathfrak{g}_{2}, \mathbf{y} \in \mathbf{g}\right)$.

Next, suppose that the Lie group $G$ has a system of one parameter subgroups $\mathbf{x}_{1}(t), \mathbf{x}_{2}(t), \mathbf{x}_{3}(t)$ such that

- each element of $G$ can be written in the form $\mathbf{x}_{1}\left(t_{1}\right) \mathbf{x}_{3}\left(t_{2}\right) \mathbf{x}_{3}\left(t_{3}\right), t_{1}, t_{2}, t_{3} \in \mathbb{R}$,

$-\left\{\mathbf{x}_{2}\left(t_{2}\right) \mathbf{x}_{3}\left(t_{3}\right) ; t_{2}, t_{3} \in \mathbb{R}\right\} \equiv G_{2}$ and $\left\{\mathbf{x}_{3}\left(t_{3}\right) ; t_{3} \in \mathbb{R}\right\} \equiv G_{3}$ are closed invariant (normal) subgroups of $G$,

- $G / G_{2}, G_{2} / G_{3}, G_{3}$ are one parameter vector groups (i.e. they are isomorphic to $\mathbb{R}$ ).

These conditions imply that each element of $G$ can be written uniquely in the form $\mathbf{x}_{1}\left(t_{1}\right) \mathbf{x}_{2}\left(t_{2}\right) \mathbf{x}_{3}\left(t_{3}\right)$, for some $t_{1}, t_{2}, t_{3} \in \mathbb{R}$. Then

- the numbers $t_{1}, t_{2}, t_{3}$ are called the (Mal'cev) 'coordinates of the second kind' of that element,

- the subgroups $\mathbf{x}_{1}(t), \mathbf{x}_{2}(t), \mathbf{x}_{3}(t)$ are called a 'system of coordinates of the second kind'.

For example, if $G=J$ and $\mathbf{g}_{1}, \mathbf{g}_{2}, \mathbf{g}_{3}$ is a system of coordinates of the first kind, then $\mathbf{x}_{i}(t) \equiv t \mathbf{g}_{i}, t \in \mathbb{R}, i=1,2,3$, is a system of coordinates of the second kind, and the converse is also true.

Lemma 1 (Mal'cev)

If a Lie group $G$ has a system of coordinates of the second kind, denoted $\mathbf{x}_{1}(t), \mathbf{x}_{2}(t), \mathbf{x}_{3}(t)$ and if a subgroup $H$ contains the elements $\mathbf{x}_{1}(1), \mathbf{x}_{2}(1), \mathbf{x}_{3}(1)$, then $H$ is uniform in $G$.

Proof See [1].

For example, the subgroup generated by $\mathbf{x}_{1}(1), \mathbf{x}_{2}(1), \mathbf{x}_{3}(1)$ is uniform.

4.2 Canonical basis of discrete groups

Elements $\mathbf{d}_{1}, \mathbf{d}_{2} \ldots \mathbf{d}_{r}$ of a nilpotent group $D$ constitute a canonical basis of $D$ if each element of $D$ can be represented in the form

$$
\mathbf{d}_{1}^{n_{1}} \mathbf{d}_{2}^{n_{2}} \ldots \mathbf{d}_{r}^{n_{r}}, \quad \text { for some } n_{i} \in \mathbb{Z}, \quad i=1,2 \ldots r
$$

and

$-\left\{\mathbf{d}_{i}^{n_{i}} \mathbf{d}_{i+1}^{n_{i+1}}, \ldots \mathbf{d}_{r}^{n_{r}} ; n_{i}, n_{i+1} \ldots n_{r} \in \mathbb{Z}\right\} \equiv D_{i}$ is an invariant subgroup of $D, i=$

- the quotient groups $D_{i} / D_{i+1}$ (where $D_{r+1}=\{\mathbf{e}\}$ ) are infinite cyclic.

These conditions imply that any element of $D$ can be written uniquely in the form (63).

Lemma 2 (Mal'cev)

Every uniform discrete subgroup $D$ of a connected simply-connected nilpotent three dimensional Lie group $G$ contains at least one canonical basis $\mathbf{d}_{1}, \mathbf{d}_{2}, \mathbf{d}_{3}$. Let $\mathbf{d}_{1}(t), \mathbf{d}_{2}(t), \mathbf{d}_{3}(t)$ be the one parameter groups passing through $\mathbf{d}_{1}, \mathbf{d}_{2}, \mathbf{d}_{3}$ such that

$$
\mathbf{d}_{i}(1)=\mathbf{d}_{i}, \quad i=1,2,3 .
$$

Then these one parameter groups provide a system of coordinates of the second kind. 
Proof See [1].

For example, if $G=J$, each uniform discrete subgroup of $J$ has a canonical basis, and corresponding systems of coordinates of the first and second kinds (via Lemmas 1 and 2 ). Also, each system of coordinates of the first kind, $\mathbf{g}_{1}, \mathbf{g}_{2}, \mathbf{g}_{3}$, induces a corresponding system of coordinates of the second kind $\mathbf{x}_{i}(t)=\mathbf{g}_{i} t, i=1,2,3, t \in \mathbb{R}$, and the subgroup generated by $\mathbf{g}_{1}, \mathbf{g}_{2}, \mathbf{g}_{3}$ is uniform.

Theorem 3 (Mal'cev)

Let $D$ and $D^{*}$ be uniform discrete subgroups of connected, simply-connected nilpotent Lie groups $G$ and $G^{*}$ respectively. Then every isomorphism between $D$ and $D^{*}$ can be uniquely extended to an isomorphism between $G$ and $G^{*}$. In particular, every automorphism of $D$ can be extended to an automorphism of $G$.

Proof See [1].

Note This theorem is proven by noting that a canonical basis $\mathbf{d}_{1}, \mathbf{d}_{2}, \mathbf{d}_{3}$ of $D$ maps to a canonical basis $\mathbf{d}_{1}^{*}, \mathbf{d}_{2}^{*}, \mathbf{d}_{3}^{*}$ of $D^{*}$ under the given isomorphism. Let the corresponding system of coordinates of the second kind (in $D$ ) be $\mathbf{d}_{1}(t), \mathbf{d}_{2}(t), \mathbf{d}_{3}(t)$ and (in $D^{*}$ ) be $\mathbf{d}_{1}^{*}(t), \mathbf{d}_{2}^{*}(t), \mathbf{d}_{3}^{*}(t)$. Then the unique extension of the given mapping to an isomorphism $G \rightarrow G^{*}$ is shown to be the map which sends $\mathbf{d}_{1}\left(t_{1}\right) \mathbf{d}_{2}\left(t_{2}\right) \mathbf{d}_{3}\left(t_{3}\right)$ to $\mathbf{d}_{1}^{*}\left(t_{1}\right) \mathbf{d}_{2}^{*}\left(t_{2}\right) \mathbf{d}_{3}^{*}\left(t_{3}\right)$.

\section{The symmetries of uniform discrete subgroups of $J$}

Let $C_{i j k}=\lambda p_{i} p_{r} \varepsilon_{r j k}$, so that via (49) $S_{a b}=\tilde{\lambda} \tilde{p}_{a} \tilde{p}_{b}$, where $\tilde{\lambda} \equiv \lambda \operatorname{det}(L), \tilde{p}_{a}=L_{a b}^{-1} p_{b}$ (recalling $\ell_{r}(\mathbf{0})=\ell_{r j}(\mathbf{0}) \mathbf{e}_{j}=L \mathbf{e}_{r}=L_{j r} \mathbf{e}_{j}$ ). Then the composition function in $J$ has the form

$$
\psi(\mathbf{x}, \mathbf{y})=\mathbf{x}+\mathbf{y}+\frac{1}{2} \lambda \mathbf{p}(\mathbf{p} \cdot \mathbf{x} \wedge \mathbf{y}), \quad \mathbf{x}, \mathbf{y} \in \mathbb{R}^{3},
$$

so that

$$
(\mathbf{x}, \mathbf{y}) \equiv \mathbf{x}^{-1} \mathbf{y}^{-1} \mathbf{x y}=\lambda \mathbf{p}(\mathbf{p} \cdot \mathbf{x} \wedge \mathbf{y})
$$

and

$$
(J, J)=\mathbb{R} \mathbf{p} .
$$

Let $D$ be a uniform discrete subgroup of $J$. Mal'cev asserts that, if $\mathbf{c}_{1}, \mathbf{c}_{2}, \mathbf{c}_{3}$ generates $D$, it provides a canonical basis of $D$ if $\mathbf{c}_{3}$ is a basis element of $D \cap(J, J)$ - I focus on this particular canonical basis. Then

$$
\mathbf{c}_{3}=\theta \mathbf{p}
$$

for some real $\theta$. Then since $\left(\mathbf{c}_{1}, \mathbf{c}_{2}\right) \in \mathbb{R} \mathbf{p}$,

$$
\left(\mathbf{c}_{1}, \mathbf{c}_{2}\right)=\mathbf{c}_{3}^{k}, \quad \text { for some } k \in \mathbb{Z} .
$$

Then from (65)

$$
\left(\mathbf{c}_{1}, \mathbf{c}_{3}\right)=\left(\mathbf{c}_{2}, \mathbf{c}_{3}\right)=\mathbf{0} .
$$

Conditions (68) and (69) are sufficient in order that $\left\{\mathbf{c}_{1}, \mathbf{c}_{2}, \mathbf{c}_{3}\right\}$ be a canonical basis of $D$. In particular, if

$$
\mathbf{c}_{1}^{\alpha_{1}} \mathbf{c}_{2}^{\beta_{1}} \mathbf{c}_{3}^{\gamma_{1}} \mathbf{c}_{1}^{\alpha_{2}} \mathbf{c}_{2}^{\beta_{2}} \mathbf{c}_{3}^{\gamma_{2}} \ldots \mathbf{c}_{1}^{\alpha_{r}} \mathbf{c}_{2}^{\beta_{r}} \mathbf{c}_{3}^{\gamma_{r}}
$$

is an arbitrary element of $D$, it can be rewritten as

$$
\mathbf{c}_{1}^{\alpha_{1}} \mathbf{c}_{2}^{\beta_{1}} \mathbf{c}_{1}^{\alpha_{2}} \mathbf{c}_{2}^{\beta_{2}} \ldots \mathbf{c}_{1}^{\alpha_{r}} \mathbf{c}_{2}^{\beta_{r}} \mathbf{c}_{3}^{\gamma_{1}+\gamma_{2}+\ldots \gamma_{r}}
$$


using (69), and by noting that

$$
\left(\mathbf{c}_{2}^{\beta}, \mathbf{c}_{1}^{\alpha}\right)=\left(\mathbf{c}_{2}, \mathbf{c}_{1}\right)^{\alpha \beta}, \mathbf{c}_{2}^{\beta} \mathbf{c}_{1}^{\alpha}=\mathbf{c}_{1}^{\alpha} \mathbf{c}_{2}^{\beta} \mathbf{c}_{3}^{-k \alpha \beta},
$$

further reshaped as

$$
\mathbf{c}_{1}^{\alpha_{1}+\alpha_{2}+\ldots \alpha_{r}} \mathbf{c}_{2}^{\beta_{1}+\beta_{2}+\ldots \beta_{r}} \mathbf{c}_{3}^{\nu},
$$

when $\nu$ can be expressed in terms of the integers $k, \alpha_{i}, \beta_{i}, \gamma_{i}, i=1,2 \ldots r$. One may check that the other conditions, required in order that $\left\{\mathbf{c}_{1}, \mathbf{c}_{2}, \mathbf{c}_{3}\right\}$ be a canonical basis of $D$, hold as well.

Now to calculate the symmetries of $D$ it is necessary first of all to digress a little by discussing general ideas regarding changes of generators in a group from the point of view of Magnus, Karrass, Solitar [12], Johnson [14]. So let $X$ be a set (which will eventually play the role of a set of generators of $D$ ), $F(X)$ be the free group with $X$ as basis, which means that: $F(X)$ consists of all 'words' in the elements of $X$ (so if, for example, $X=\left\{x_{1}, x_{2}, x_{3}\right\}$, the words of $F(X)$ have the form $\omega=x_{1}^{\alpha_{1}} x_{2}^{\beta_{1}} x_{3}^{\gamma_{1}} x_{1}^{\alpha_{2}} x_{2}^{\beta_{2}} x_{3}^{\gamma_{2}} \ldots x_{1}^{\alpha_{r}} x_{2}^{\beta_{r}} x_{3}^{\gamma_{r}}$ for some integer $r$, integers $\alpha_{i}, \beta_{i}, \gamma_{i}$, $i=1,2, \ldots r)$. The group operation in $F(X)$ is juxtaposition of words, with terms of the form $x_{i} x_{i}^{-1}, x_{i}^{-1} x_{i}$ 'cancelled' in any product of words. In fact, confine attention to the case $X=\left\{x_{1}, x_{2}, x_{3}\right\}$. Then a 'free substitution' of $F(X)$ is a replacement of the elements $x_{1}, x_{2}, x_{3}$ by words $\omega_{1}\left(x_{1}, x_{2}, x_{3}\right), \omega_{2}\left(x_{1}, x_{2}, x_{3}\right), \omega_{3}\left(x_{1}, x_{2}, x_{3}\right) \in F(X)$ such that these words are also a basis of $F(X)$. (For example, one may take $\omega_{1}=$ $\left.x_{1}, \omega_{2}=x_{2}, \omega_{3}=x_{1} x_{3}\right)$. This implies, in particular, that each $x_{i}, i=1,2,3$, may be written as a word in $\omega_{1}, \omega_{2}, \omega_{3}$ (thus, in the example, $x_{1}=\omega_{1}, x_{2}=\omega_{2}, x_{3}=$ $\left.\omega_{1}^{-1} \omega_{3}\right)$, and this fact alone is sufficient that $\left\{\omega_{1}, \omega_{2}, \omega_{3}\right\}$ is a basis of $F(X)$. Also, a free substitution gives rise to a mapping which sends any $\omega\left(x_{1}, x_{2}, x_{3}\right) \in F(X)$ to $\omega\left(\omega_{1}\left(x_{1}, x_{2}, x_{3}\right), \omega_{2}\left(x_{1}, x_{2}, x_{3}\right), \omega_{3}\left(x_{1}, x_{2}, x_{3}\right)\right) \in F(X)$, and it is a fact that this mapping is an automorphism of $F(X)$.

Thus the free substitutions represent changes in the set of generators of a free group. It is important to note that such changes of generators, if applied to a given group $D$ with generators $\left\{x_{1}, x_{2}, x_{3}\right\}$, do not generally provide automorphisms of $D$. [For example, the uniform discrete group $D \subseteq J$ has generators $\mathbf{c}_{1}, \mathbf{c}_{2}, \mathbf{c}_{3}$ with $\left(\mathbf{c}_{1}, \mathbf{c}_{2}\right)=$ $\mathbf{c}_{3}^{k},\left(\mathbf{c}_{1}, \mathbf{c}_{3}\right)=\left(\mathbf{c}_{2}, \mathbf{c}_{3}\right)=\mathbf{0}$. Suppose that $\boldsymbol{\phi}: D \rightarrow D$ is an automorphism, then it is easy to show that $\left(\phi\left(\mathbf{c}_{1}\right), \phi\left(\mathbf{c}_{2}\right)\right)=\left(\phi\left(\mathbf{c}_{3}\right)\right)^{k},\left(\phi\left(\mathbf{c}_{1}\right), \phi\left(\mathbf{c}_{3}\right)\right)=\left(\phi\left(\mathbf{c}_{2}\right), \phi\left(\mathbf{c}_{3}\right)\right)=\mathbf{0}$. One obtains a contradiction if one presumes that $\phi$ is the free substitution $\phi\left(\mathbf{c}_{1}\right)=$ $\mathbf{c}_{1}, \phi\left(\mathbf{c}_{2}\right)=\mathbf{c}_{2}, \phi\left(\mathbf{c}_{3}\right)=\mathbf{c}_{1} \mathbf{c}_{3}$.] The condition that a free substitution may be associated with an automorphism of $D$ is provided by a lemma of Magnus, Karrass, Solitar [12], Johnson [14]:

\section{Lemma 4}

Let $\omega\left(\mathbf{c}_{1}, \mathbf{c}_{2}, \mathbf{c}_{3}\right) \equiv \mathbf{c}_{1}^{\alpha_{1}} \mathbf{c}_{2}^{\beta_{1}} \mathbf{c}_{3}^{\gamma_{1}} \mathbf{c}_{1}^{\alpha_{2}} \mathbf{c}_{2}^{\beta_{2}} \mathbf{c}_{3}^{\gamma_{2}} \ldots \mathbf{c}_{1}^{\alpha_{r}} \mathbf{c}_{2}^{\beta_{r}} \mathbf{c}_{3}^{\gamma_{r}}$, where $\alpha_{i}, \beta_{i}, \gamma_{i}, i=1,2, \ldots r$ are integers, be any word in the generators $\mathbf{c}_{1}, \mathbf{c}_{2}, \mathbf{c}_{3}$ of $D$ such that

$$
\omega\left(\mathbf{c}_{1}, \mathbf{c}_{2}, \mathbf{c}_{3}\right)=\mathbf{0}
$$

$\mathbf{0}$ the group identity. Then a free substitution $\phi$ extends to an automorphism of $D$ if and only if

$$
\omega\left(\phi\left(\mathbf{c}_{1}\right), \phi\left(\mathbf{c}_{2}\right), \phi\left(\mathbf{c}_{3}\right)\right)=\mathbf{0}, \quad \text { and } \quad \omega\left(\phi^{-1}\left(\mathbf{c}_{1}\right), \phi^{-1}\left(\mathbf{c}_{2}\right), \phi^{-1}\left(\mathbf{c}_{3}\right)\right)=\mathbf{0} .
$$

where $\phi^{-1}$ is the free substitution that maps $\phi\left(\mathbf{c}_{i}\right)$ to $\mathbf{c}_{i}$. 
Proof

See $[12],[14]$.

This lemma allows the automorphisms of $D$ to be calculated explicitly, for all relations in $D$ of the form $\omega\left(\mathbf{c}_{1}, \mathbf{c}_{2}, \mathbf{c}_{3}\right)=\mathbf{e}$ follow from (68) and (69):

$$
\left(\mathbf{c}_{1}, \mathbf{c}_{2}\right)=\mathbf{c}_{3}^{k},\left(\mathbf{c}_{1}, \mathbf{c}_{3}\right)=\left(\mathbf{c}_{2}, \mathbf{c}_{3}\right)=\mathbf{0} .
$$

Thus, if a free substitution $\phi$ is to be an automorphism of $D$, then according to Lemma $4,(73)$ is to hold with $\mathbf{c}_{i}$ replaced by $\phi\left(\mathbf{c}_{i}\right), i=1,2,3$, and with $\mathbf{c}_{i}$ replaced by $\phi^{-1}\left(\mathbf{c}_{i}\right)$, $i=1,2,3$. So, since $\phi: D \rightarrow D$, and $\left\{\mathbf{c}_{1}, \mathbf{c}_{2}, \mathbf{c}_{3}\right\}$ is a canonical basis of $D$,

$$
\phi\left(\mathbf{c}_{i}\right)=\mathbf{c}_{1}^{\alpha_{i}} \mathbf{c}_{2}^{\beta_{i}} \mathbf{c}_{3}^{\gamma_{i}}, \quad i=1,2,3, \quad \text { no summation over } i,
$$

for integers $\alpha_{i}, \beta_{i}, \gamma_{i}, i=1,2,3$. Likewise

$$
\phi^{-1}\left(\mathbf{c}_{i}\right)=\mathbf{c}_{1}^{p_{i}} \mathbf{c}_{2}^{q_{i}} \mathbf{c}_{3}^{r_{i}}, \quad i=1,2,3, \quad \text { no summation over } i,
$$

for integers $p_{i}, q_{i}, r_{i}, i=1,2,3$. One calculates that, for example,

$$
\left(\phi\left(\mathbf{c}_{1}\right), \phi\left(\mathbf{c}_{2}\right)\right)=\mathbf{c}_{3}^{k\left(\alpha_{1} \beta_{2}-\alpha_{2} \beta_{1}\right)}=\phi\left(\mathbf{c}_{3}^{k}\right)=\left(\mathbf{c}_{1}^{\alpha_{3}} \mathbf{c}_{2}^{\beta_{3}} \mathbf{c}_{3}^{\gamma_{3}}\right)^{k} .
$$

Using (70), it follows that

$$
\alpha_{3}=\beta_{3}=0, \quad \gamma_{3}=\alpha_{1} \beta_{2}-\alpha_{2} \beta_{1},
$$

and a similar calculation for $\left(\phi^{-1}\left(\mathbf{c}_{1}\right), \phi^{-1}\left(\mathbf{c}_{2}\right)\right)$ gives

$$
p_{3}=q_{3}=0, \quad r_{3}=p_{1} q_{2}-p_{2} q_{1} \text {. }
$$

Next $\phi^{-1}\left(\phi\left(\mathbf{c}_{i}\right)\right)=\mathbf{c}_{i}$ gives

$$
\left(\mathbf{c}_{1}^{\alpha_{1}} \mathbf{c}_{2}^{\beta_{1}} \mathbf{c}_{3}^{\gamma_{1}}\right)^{p_{i}}\left(\mathbf{c}_{1}^{\alpha_{2}} \mathbf{c}_{2}^{\beta_{2}} \mathbf{c}_{3}^{\gamma_{2}}\right)^{q_{i}}\left(\mathbf{c}_{1}^{\alpha_{3}} \mathbf{c}_{2}^{\beta_{3}} \mathbf{c}_{3}^{\gamma_{3}}\right)^{r_{i}}=\mathbf{c}_{i}, i=1,2,3 \text {, no summation over } i \text {. }
$$

Manipulation of (79), using (29) and (73), gives

$$
\left(\begin{array}{ll}
p_{1} & p_{2} \\
q_{1} & q_{2}
\end{array}\right)\left(\begin{array}{ll}
\alpha_{1} & \alpha_{2} \\
\beta_{1} & \beta_{2}
\end{array}\right)=\left(\begin{array}{ll}
1 & 0 \\
0 & 1
\end{array}\right)
$$

and

$$
r_{3} \gamma_{3}=1,
$$

with no constraint on $\gamma_{1}, \gamma_{2}$ (other than that they are integers). Then $r_{1}, r_{2}$ are determined in terms of the remaining parameters. It follows that

$$
\left.\begin{array}{rl}
\alpha_{1} \beta_{2}-\alpha_{2} \beta_{1} & =\varepsilon, \quad \text { where } \varepsilon= \pm 1, \\
p_{1} q_{2}-p_{2} q_{1} & =\varepsilon, \\
\gamma_{3}=r_{3} & =\varepsilon .
\end{array}\right\}
$$

Thus the automorphisms of $D$ have the form $\phi: D \rightarrow D$ where

$$
\phi\left(\mathbf{c}_{i}\right)=\mathbf{c}_{1}^{\alpha_{i}} \mathbf{c}_{2}^{\beta_{i}} \mathbf{c}_{3}^{\gamma_{i}}, \quad i=1,2,3, \quad \text { no summation on } i,
$$


and

$$
\left(\begin{array}{lll}
\alpha_{1} & \alpha_{2} & \alpha_{3} \\
\beta_{1} & \beta_{2} & \beta_{3} \\
\gamma_{1} & \gamma_{2} & \gamma_{3}
\end{array}\right)=\left(\begin{array}{lll}
\alpha_{1} & \alpha_{2} & 0 \\
\beta_{1} & \beta_{2} & 0 \\
\gamma_{1} & \gamma_{2} & \varepsilon
\end{array}\right)
$$

where $\varepsilon= \pm 1, \alpha_{1} \beta_{2}-\alpha_{2} \beta_{1}=\varepsilon, \gamma_{1}$ and $\gamma_{2}$ are arbitrary integers. In particular,

$$
\left(\begin{array}{cc}
\alpha_{1} & \alpha_{2} \\
\beta_{1} & \beta_{2}
\end{array}\right) \in G L_{2}(\mathbb{Z})
$$

Note that

$$
\left(\begin{array}{rr}
-1 & 0 \\
0 & 1
\end{array}\right),\left(\begin{array}{ll}
1 & 1 \\
0 & 1
\end{array}\right),\left(\begin{array}{rr}
0 & -1 \\
1 & 0
\end{array}\right) \quad \text { generate } G L_{2}(\mathbb{Z})
$$

Therefore matrices of the form on the right hand side of (83) are generated by

$$
\left(\begin{array}{rrr}
-1 & 0 & 0 \\
0 & 1 & 0 \\
0 & 0 & -1
\end{array}\right),\left(\begin{array}{lll}
1 & 1 & 0 \\
0 & 1 & 0 \\
0 & 0 & 1
\end{array}\right),\left(\begin{array}{rrr}
0 & -1 & 0 \\
1 & 0 & 0 \\
0 & 0 & 1
\end{array}\right),\left(\begin{array}{lll}
1 & 0 & 0 \\
0 & 1 & 0 \\
1 & 0 & 1
\end{array}\right),\left(\begin{array}{lll}
1 & 0 & 0 \\
0 & 1 & 0 \\
0 & 1 & 1
\end{array}\right) \text {. }
$$

Correspondingly, the automorphisms of $D$ include the mappings:

$$
\begin{array}{lll}
\text { - } \mathbf{c}_{1} \rightarrow \mathbf{c}_{1}^{-1}, & \mathbf{c}_{2} \rightarrow \mathbf{c}_{2}, & \mathbf{c}_{3} \rightarrow \mathbf{c}_{3}^{-1}, \\
\text { - } \mathbf{c}_{1} \rightarrow \mathbf{c}_{1}, & \mathbf{c}_{2} \rightarrow \mathbf{c}_{1} \mathbf{c}_{2}, & \mathbf{c}_{3} \rightarrow \mathbf{c}_{3} \\
\text { - } \mathbf{c}_{1} \rightarrow \mathbf{c}_{2}, & \mathbf{c}_{2} \rightarrow \mathbf{c}_{1}^{-1}, & \mathbf{c}_{3} \rightarrow \mathbf{c}_{3} \\
\text { - } \mathbf{c}_{1} \rightarrow \mathbf{c}_{1} \mathbf{c}_{3}, & \mathbf{c}_{2} \rightarrow \mathbf{c}_{2}, & \mathbf{c}_{3} \rightarrow \mathbf{c}_{3} \\
\text { - } \mathbf{c}_{1} \rightarrow \mathbf{c}_{1}, & \mathbf{c}_{2} \rightarrow \mathbf{c}_{2} \mathbf{c}_{3}, & \mathbf{c}_{3} \rightarrow \mathbf{c}_{3}
\end{array}
$$

It can be shown that these particular automorphisms generate the (group of) automorphisms of $D$, and that each mapping (87) provides a free substitution of $F\left(\left\{\mathbf{c}_{1}, \mathbf{c}_{2}, \mathbf{c}_{3}\right\}\right)$. Thus, (82), (83) catalogue the automorphisms of $D$ which derive from free substitutions of $F\left(\left\{\mathbf{c}_{1}, \mathbf{c}_{2}, \mathbf{c}_{3}\right\}\right)$. Also, each of the automorphisms (82), (83) extends to an automorphism of $J$, according to theorem 3 . Then, by the note which follows that theorem, put $\phi\left(\mathbf{c}_{i}\right)=\mathbf{c}_{i}^{*}, i=1,2,3$, and define $\phi^{J}: J \rightarrow J$ by

$$
\phi^{J}\left(\left(t_{1} \mathbf{c}_{1}\right)\left(t_{2} \mathbf{c}_{2}\right)\left(t_{3} \mathbf{c}_{3}\right)\right)=\left(t_{1} \mathbf{c}_{1}^{*}\right)\left(t_{2} \mathbf{c}_{2}^{*}\right)\left(t_{3} \mathbf{c}_{3}^{*}\right),
$$

noting that the one parameter groups in $J$ are straight lines. But

$$
\begin{aligned}
\left(t_{1} \mathbf{c}_{1}\right)\left(t_{2} \mathbf{c}_{2}\right)\left(t_{3} \mathbf{c}_{3}\right) & =\left(t_{1} \mathbf{c}_{1}+t_{2} \mathbf{c}_{2}+\frac{1}{2}\left[t_{1} \mathbf{c}_{1}, t_{2} \mathbf{c}_{2}\right]\right)\left(t_{3} \mathbf{c}_{3}\right) \\
& =t_{1} \mathbf{c}_{1}+t_{2} \mathbf{c}_{2}+\left(t_{3}+\frac{1}{2} k t_{1} t_{2}\right) \mathbf{c}_{3},
\end{aligned}
$$

by virtue of (39) (or (64), (68), (69)), noting that $\mathbf{c}_{3}^{k}=k \mathbf{c}_{3}$. Likewise

$$
\left(t_{1} \mathbf{c}_{1}^{*}\right)\left(t_{2} \mathbf{c}_{2}^{*}\right)\left(t_{3} \mathbf{c}_{3}^{*}\right)=t_{1} \mathbf{c}_{1}^{*}+t_{2} \mathbf{c}_{2}^{*}+\left(t_{3}+\frac{1}{2} k t_{1} t_{2}\right) \mathbf{c}_{3}^{*},
$$

since the automorphism $\phi$ preserves multiplication and the bracket operations. Hence $\phi^{J}\left(\alpha_{i} \mathbf{c}_{i}\right)=\alpha_{i} \phi^{J}\left(\mathbf{c}_{i}\right)=\alpha_{i} \phi\left(\mathbf{c}_{i}\right)$, and $\phi^{J}$ is a linear mapping, as proven in section 2.4. The linear mapping is uniquely determined by its values on a (canonical) basis of $D$ : let

$$
\phi^{J}\left(\alpha_{i} \mathbf{c}_{i}\right)=\alpha_{i} \phi\left(\mathbf{c}_{i}\right)=\alpha_{i} L_{j i}^{\mathbf{c}} \mathbf{c}_{j},
$$


so that $L_{j i}^{\mathbf{c}}$ are the components of this mapping with respect to the basis $\mathbf{c}_{1}, \mathbf{c}_{2}, \mathbf{c}_{3}$. Then

$$
\begin{aligned}
& L \mathbf{c}_{i} \equiv L_{j i}^{\mathbf{c}} \mathbf{c}_{j}=\mathbf{c}_{i}^{*}=c_{1}^{\alpha_{i}} c_{2}^{\beta_{i}} c_{3}^{\gamma_{i}}=\alpha_{i} \mathbf{c}_{1}+\beta_{i} \mathbf{c}_{2}+\left(\gamma_{i}+\frac{1}{2} k \alpha_{i} \beta_{i}\right) \mathbf{c}_{3}, \\
& i=1,2,3, \quad \text { no summation on } i \text {, }
\end{aligned}
$$

and so

$$
\left(L_{j i}^{\mathbf{c}}\right)=\left(\begin{array}{ccc}
\alpha_{1} & \alpha_{2} & 0 \\
\beta_{1} & \beta_{2} & 0 \\
\gamma_{1}+\frac{1}{2} \alpha_{1} \beta_{1} k & \gamma_{2}+\frac{1}{2} \alpha_{2} \beta_{2} k & \varepsilon
\end{array}\right)
$$

where $\alpha_{1} \beta_{2}-\alpha_{2} \beta_{1}=\varepsilon= \pm 1, \gamma_{1}, \gamma_{2}$ are arbitrary integers.

\section{Elastic and inelastic symmetries of sets of points associated with a defective crystal}

The focus of the paper has been on crystals with ddt $S_{a b}=\lambda p_{a} p_{b}, a, b=1,2,3$, where each of the elements $S_{a b}$ is rational and where one can accordingly assume that $p_{1}, p_{2}, p_{3}$ are relatively prime integers and $\lambda$ is rational. Then $\mathbf{p} \equiv\left(p_{1}, p_{2}, p_{3}\right)$ is uniquely determined by $S$. Also, attention has been confined to discrete subgroups $D \subseteq$ $J$ with canonical basis $\mathbf{c}_{1}, \mathbf{c}_{2}, \mathbf{c}_{3}$, with $\mathbf{c}_{3}$ parallel to $\mathbf{p},\left(\mathbf{c}_{1}, \mathbf{c}_{2}\right)=\mathbf{c}_{3}^{k}$ for some integer $\bar{k}$. The intention is to use the apparatus developed in the body of the paper to motivate the adoption of appropriate symmetry properties for potential energy functions relevant to the mechanics of defective crystals, specifically functions which are to have the form

$$
w=w\left(\left\{\mathbf{e}_{a}\right\}, S\right)
$$

when $\left\{\mathbf{e}_{a}\right\}=\left\{\mathbf{e}_{1}, \mathbf{e}_{2}, \mathbf{e}_{3}\right\}$ represent the local texture of the crystal. The idea that this function represents the energy of the physical points that correspond to elements of the discrete subgroup $D \subseteq J$ which has generators $\mathbf{e}_{1}, \mathbf{e}_{2}, \mathbf{e}_{3}$, where $J$ is the canonical Lie group whose Lie algebra has structure constants determined by $S$ via (49), with $\boldsymbol{\ell}_{a}(\mathbf{0})=\mathbf{e}_{a}, a=1,2,3$. I focus on the case where $\left\{\mathbf{e}_{a}\right\}$ is chosen to be a canonical basis of $D$, so $\mathbf{e}_{a}=\mathbf{c}_{a}, a=1,2,3$, and (73) holds. If $\phi: D \rightarrow D$ is an automorphism, then $\left\{\phi\left(\mathbf{c}_{a}\right)\right\} \equiv\left\{\mathbf{c}_{a}^{*}\right\}$ is also a canonical basis of $D . \phi$ is well-defined, it extends to an automorphism of $J$, and that extension represents a homogeneous elastic deformation of the defective crystal. Thus the lattice vector fields $\boldsymbol{\ell}_{a}^{*}(\cdot), a=1,2,3$, which are such that $\boldsymbol{\ell}_{a}^{*}(\mathbf{0})=\mathbf{c}_{a}^{*}, a=1,2,3$, are obtained from the fields $\boldsymbol{\ell}_{a}(\cdot), a=1,2,3$, by elastic deformation, and it follows that if these fields have ddt $S^{*}$, then $S^{*}=S$ by the elastic invariance of the ddt. If one accepts that the function $w$ depends just on the set of points that $D$ represents, then

$$
w\left(\left\{\mathbf{c}_{a}\right\}, S\right)=w\left(\left\{\phi\left(\mathbf{c}_{a}\right)\right\}, S\right),
$$

whenever $\phi$ is an automorphism of $D$. I shall consider elsewhere how to extend (93) in the case where the first argument of $w$ is not a canonical basis of $D$.

Relation (93) identifies the symmetries of $D$ that relate to well defined mappings $\phi: D \rightarrow D$ that preserve the group structure. Next, I show that there are other types of symmetry, that do not preserve the group structure, but nevertheless preserve the set of points that $D$ represents. Note that from (68), (69), using the identification of Lie bracket and group commutator, $\left[\mathbf{c}_{1}, \mathbf{c}_{3}\right]=\left[\mathbf{c}_{2}, \mathbf{c}_{3}\right]=\mathbf{0},\left[\mathbf{c}_{1}, \mathbf{c}_{2}\right]=k \mathbf{c}_{3}$, for some 
integer $k$, where $\mathbf{c}_{1}, \mathbf{c}_{2}, \mathbf{c}_{3}$ is a canonical basis of $D \subseteq J$. Also, from (13), (16), the structure constants $C_{i j k}$ with respect to basis $\mathbf{c}_{1}, \mathbf{c}_{2}, \mathbf{c}_{3}$, satisfy $\left[\mathbf{c}_{\beta}, \mathbf{c}_{\gamma}\right]=C_{\alpha \beta \gamma} \mathbf{c}_{\alpha}$, $\alpha, \beta, \gamma=1,2,3$. Hence $C_{\alpha \beta \gamma}=k \varepsilon_{\beta \gamma 3} \delta_{\alpha 3}$ and from (52), $S_{33}=k, S_{a b}=0$ otherwise. So from (63),

$$
D=\left\{\mathbf{x}: \mathbf{x}=\mathbf{c}_{1}^{n_{1}} \mathbf{c}_{2}^{n_{2}} \mathbf{c}_{3}^{n_{3}}, \quad n_{i} \in \mathbb{Z}, \quad i=1,2,3\right\},
$$

and recalling (89)

$$
\mathbf{c}_{1}^{n_{1}} \mathbf{c}_{2}^{n_{2}} \mathbf{c}_{3}^{n_{3}}=n_{1} \mathbf{c}_{1}+n_{2} \mathbf{c}_{2}+\left(n_{3}+\frac{1}{2} k n_{1} n_{2}\right) \mathbf{c}_{3} .
$$

It follows immediately that, if $k$ is even,

$$
D=\left\{\mathbf{x}: \mathbf{x}=n_{i} \mathbf{c}_{i}, \quad n_{i} \in \mathbb{Z}, \quad i=1,2,3\right\} .
$$

Thus, $D$ is a simple lattice with basis $\mathbf{c}_{1}, \mathbf{c}_{2}, \mathbf{c}_{3}$ in the case that $S_{33}$ is an even integer, $S_{a b}=0$ otherwise. Therefore, if one accepts that the energy function $w$ depends just on the set of points that $D$ represents,

$$
w\left(\left\{\mathbf{c}_{a}\right\},\left(k \delta_{3 a} \delta_{3 b}\right)\right)=w\left(\left\{\mathbf{c}_{a}\right\}, 0\right), \quad k \text { even, }
$$

and this is an inelastic symmetry of the energy function, since the ddt is non zero on the left hand side of (97), zero on the right hand side, and the ddt is an elastic invariant.

Relation (97) expresses the fact that a simple lattice has an infinite number of different representations as a discrete structure associated with a defective crystal. It is a fascinating problem, to investigate how the paths and circuits in one description of this structure are related to the paths and circuits in a different description - however that task seems to require some subtleties additional to the material presented here, so I leave it to future work.

\section{References}

1. A. Mal'cev, On a class of homogeneous spaces, Isvestiya Akad. Nauk SSSR Ser., Mat 13, pp 9-32 (1949). Am. Math. Soc. Translation 39 (1949).

2. P. Cermelli and G. P. Parry, The structure of uniform discrete defective crystals, Continuum Mech. Thermodyn., 18, 2006, 47-61.

3. M. Elzanowski and G. P. Parry, Material symmetry in a theory of continuously defective crystals, J. Elasticity, 74, 215-237 (2004).

4. G. P. Parry, Group properties of defective crystal structures, Mathematics and Mechanics of Solids, 8, 2003, 515-537.

5. G. P. Parry, Rotaitonal symmetries of crystals with defects, J. Elast. 94 147-166 (2009).

6. C. Davini, A proposal for a continuum theory of defective crystals. Arch. Rational Mech. Anal., 96, 1986, 295-317.

7. Davini, C., Parry, G.P., On defect preserving deformations in crystals, Int. J. of Plasticity 5, 337-369 (1989)

8. Davini, C., Parry, G.P., A complete list of invariants for defective crystals, Proc. R. Soc. Lond. A 432, 341-365 (1991).

9. G. P. Parry, Symmetries of continuously defective crystals, in Mathematical modelling of bodies with complicated bulk and boundary behaviour, ed. M. Silhavý, Quaderni di Mathematica 20, Dipartimento di Matematica della Seconda Università di Napoli, 2007, $135-158$.

10. V. V. Gorbatsevich, A. L. Onishchik, E. B. Vinberg, Foundations of Lie theory and Lie transformation groups, Springer-Verlag, Berlin, Heidelberg (1997). 
11. P. Hall, A contribution to the theory of groups of prime power order, Proc. London. Math. Soc., Ser 2, 36, 29-95 (1933).

12. W. Magnus, A. Karrass, D. Solitar, Combinatorial Group Theory, Dover, New York (1976).

13. V. S. Varadarajan, Lie groups, Lie algebras, and their representations, Prentice-Hall, Englewood Cliffs (1974).

14. D. L. Johnson, Presentations of groups, London Mathematical Society Student Texts 15, 2nd Edition, Cambridge University Press (1997). 